\title{
Controlled Lithium Deposition
}

\author{
Simeng Zhang ${ }^{1,2}$, Gaojing Yang ${ }^{1,3}$, Xiaoyun $L^{1,2}$, Yejing $L^{1}{ }^{1,3}$, Zhaoxiang Wang ${ }^{1,2,3 *}$ and \\ Liquan Chen ${ }^{1}$
}

${ }^{1}$ Key Laboratory for Renewable Energy, Beijing Key Laboratory for New Energy Materials and Devices, Institute of Physics, Chinese Academy of Sciences, Beijing, China, ${ }^{2}$ College of Materials Science and Opto-Electronic Technology, University of Chinese Academy of Sciences, Beijing, China, ${ }^{3}$ School of Physical Sciences, University of Chinese Academy of Sciences, Beijing, China

Lithium metal is a promising anode material for its low redox potential and high theoretical specific capacity. However, the commercial application of the lithium metal anode is hindered with safety concerns arising from the uncontrolled growth of the lithium dendrites and significant volume variation during the lithium plating and stripping processes. Modification to the current collector is effective in tailoring the morphology of the deposited lithium and improving the cycling performance of the lithium metal batteries This review summarizes at first the global research advances in the structural design and the selection of the current collectors and their textures. It then presents some of our efforts in realizing controlled lithium deposition by designing current collectors in three aspects, lithium deposition induced by the micro-to-nano structures, lithiophilic alloys and iron

OPEN ACCESS

Edited by:

Zonghai Chen,

Argonne National Laboratory (DOE), United States

Reviewed by:

Xiangbo Meng,

University of Arkansas, United States Yongzhu Fu,

Zhengzhou University, China

*Correspondence:

Zhaoxiang Wang,

zxwang@iphy.ac.cn

Specialty section:

This article was submitted to Electrochemical Energy Conversion and Storage,

a section of the journal Frontiers in Energy Research

Received: 16 December 2021 Accepted: 05 January 2022 Published: 03 February 2022

Citation:

Zhang S, Yang G, Li X, Li Y, Wang Z and Chen $L$ (2022) Controlled Lithium Deposition. Front. Energy Res. 10:837071. doi: 10.3389/fenrg.2022.837071 carbides. Finally, conclusions and prospects are made for the further research of the current collectors.

\section{Keywords: lithium metal battery, current collector, lithium dendrites, structure design, materials selection}

\section{INTRODUCTION}

Lithium metal anode, known for its high theoretical capacity and low redox potential, has received considerable interest for the high energy-density batteries (Tarascon and Armand, 2001; Zhang et al., 2021a). Efficient and reversible lithium plating/stripping is the basis of various secondary batteries such as the lithium-sulfur ( $\mathrm{Li}-\mathrm{S})$, lithium-oxygen $\left(\mathrm{Li}-\mathrm{O}_{2}\right)$ and all-solid-state lithium batteries. However, the commercial application of the lithium metal anode is impeded with the uncontrolled growth of the lithium dendrites (Xu et al., 2014).

The growth of the lithium dendrites results in a series of severe problems. 1. The dendrite may impale the separator and result in short circuit and safety concerns. 2 . The lithium dendrites with a large surface area enhance the detrimental side reactions and formation of "dead" lithium, thereby leading to a short lifespan, large polarization and low energy conversion efficiency (Zhang S. et al., 2020). 3. In comparison with the intercalation anodes such as graphite (Jiang et al., 2021) and silicon (Hu et al., 2021; Tan Darren et al., 2021), the lithium plating/stripping is host-less and accompanied with large volume variation. The dendrite growth engenders harsher volume change (Cheng et al., 2017). Therefore, it is critical for the safe and efficient operation of the lithium metal batteries (LMB) to control the growth of the lithium metal, including the lithium nucleation location, lithium growth direction, and the morphology of the deposited lithium.

Various methods have been developed to realize the controlled deposition of the lithium metal, including optimizing the electrolytes (Yang et al., 2019a; Zhang S. et al., 2020; Zhang et al., 2021a; Yang et al., 2021b), applying the solid electrolytes, designing the current collectors (Yang et al., 2019c) and building artificial solid electrolyte interface (SEI) layers. The electrolyte additives such as fluorinated ethylene carbonate (FEC) (Zhang X.-Q. et al., 2017; Hou et al., 2019; Lin and Zhao, 2020), 
$\mathrm{LiNO}_{3}$ (Zhang et al., 2021a; May et al., 2021; Piao et al., 2021; Wahyudi et al., 2021) and vinylene carbonate (VC) (Kuwata et al., 2016; Xu Y. et al., 2020) are beneficial for forming stable SEI layers and inhibiting the growth of the lithium dendrites. The application of the gel electrolytes with high conductivity or solid electrolytes with high mechanical strength to replace the conventional liquid electrolytes also reached great achievements in improving the cycling stability. Many inorganic components such as $\mathrm{LiF}$ (Yuan et al., 2019), $\mathrm{Li}_{3} \mathrm{~N}$ (Dong et al., 2020), $\mathrm{Al}_{2} \mathrm{O}_{3}$ (Jing et al., 2015), organic coatings such as poly (vinylidene difluoride) (PVDF) (Dong et al., 2019) film and organicinorganic composite layers were used as artificial protective layers to inhibit the side reactions between lithium and electrolytes. Construction of the appropriate current collectors such as lithiophilic alloy current collectors (Li W. et al., 2019; Liu S. et al., 2019; Wan et al., 2020; Ye et al., 2020) reduces the lithium nucleation barrier, assists the uniform deposition of lithium and suppresses the growth of the lithium dendrites.

This review is focused on the construction of the current collectors, including designing the structure and selecting the texture of the current collectors. After a brief introduction about the global research advances for the controlled lithium deposition, this review presents some of our recent work on designing the current collectors to achieve the controlled deposition of lithium metal in three aspects: selective lithium deposition on micro-nano patterns, lithium deposition induced by lithiophilic alloys and underpotential lithium deposition in carbon nanotube (CNT) cavities. Finally, we will discuss some new insights and future directions associated with the controlled lithium deposition.

\section{RECENT RESEARCH ADVANCES}

The current collector plays a vital role in the lithium metal deposition process, and its characteristics significantly affect the morphology and uniformity of the deposited lithium. An appropriately designed current collector can adjust the lithium plating/stripping behavior to inhibit the growth of the lithium dendrites and minimize the volume change of the electrode during repeated cycling. There are two aspects for the design, structure and texture of the current collectors.

\subsection{The Structural Design of the Current Collector}

The inhomogeneous distribution of the local current density and the electrolyte concentration is believed to be the main reason for the uncontrolled growth of the Li dendrites. The porous current collectors can modulate the distribution of the electric field in the electrolyte through its conductive surface structure, and adjust the lithium ion concentration gradient in the electrolyte by reducing the local current density near the anode surface. Therefore, specifically designing the structure of the current collector is an effective approach to simultaneously regulating the lithium deposition and controlling both the position and morphology of the deposited lithium.

\subsubsection{Micro/nano Patterned Current Collectors}

Designing the current collector with appropriate micro-/nanopores is a promising strategy to well control the position and growth direction of the lithium metal and suppress the growth of the lithium dendrites (Zhang Y. et al., 2017; Wang A. et al., 2018; Zou et al., 2018; Zhai et al., 2020). The lithium metal itself can be designed as micro- or nanostructured frameworks. Ryou et al. (Ryou et al., 2015) fabricated some surface patterns on the lithium foil by micro-needle pre-treatment technique to direct and control the lithium deposition. As the preferential sites for the controlled lithium plating were generated by the rolling process, the as-obtained surface structure can conveniently suppress the lithium dendrite formation during the lithium deposition. Other methods for making surface-patterned lithium metal include simple stamping (Park et al., 2016) and nano imprinting (Li et al., 2018). However, the stability of the micro-nano structure on the lithium metal is still problematic. The micro/nanopores on the surface of the lithium metal can guide lithium to deposit in the pores or pits during the first lithium plating, but the micro-nano structure is damaged or even destructed after the lithium dissolution. The preparation of micro-nano structures on other current collectors can ensure their stability during cycling. Wang et al. (Wang S.-H. et al., 2017) chose $\mathrm{Cu}$ foil with micro-sized cylindrical pits as the current collector and successfully deposited lithium into the channels by regulating the current density distribution. When the $\mathrm{Cu}$ current collector with deposited lithium was assembled with a $\mathrm{LiFePO}_{4}$ cathode, the battery exhibited a capacity retention of $90 \%$ after 100 cycles, in contrast with the $80 \%$ capacity retention of a planar $\mathrm{Cu}$ foil. It is worth noting that better cycle performances can be obtained by tailoring the geometric parameters of the micro/nano structure (Chen K.-H. et al., 2019). Chen et al. (Chen K.-H. et al., 2019) improved the electrochemical performance by optimizing the pore diameter, spacing and length of a three-dimensional (3D) $\mathrm{Cu}$ current collector. They found that pores with small diameters cannot ensure the selective lithium deposition therein, while large pores cannot regulate the morphology of the deposited lithium. Wang et al. (Wang S.-H. et al., 2017) simulated the current density distribution and proved that the current density within the micro-channels is obviously larger than on the upper in the case of the porous $\mathrm{Cu}$ current collector, thus contributing to the preferential nucleation of lithium inside the openings of the channels. These prove the feasibility of using micro-nano pores to adjust the lithium deposition position.

\subsubsection{The 3D Porous Current Collectors}

The pores that can guide lithium deposition are not limited to the artificially prepared micro-pores. Various natural nano-pores such as the gaps or cracks between nanowires (Lu et al., 2017; Yan K. et al., 2018; Shang et al., 2020) and nanoparticles (Wang Y. et al., 2018; Ma et al., 2019; Qiu et al., 2019) were also proved feasible in controlling the location of the lithium deposition. The 3D current collectors composed of $\mathrm{Cu}$ nanofibers (Yang et al., 2015) and nanoparticles (Ma et al., 2019) were used to effectively improve the lithium plating/stripping performance. Guo et al. (Yang et al., 2015) compared the lithium deposition behaviors on 
a planar $\mathrm{Cu}$ foil and on a 3D $\mathrm{Cu}$ foil composed of bundles of $\mathrm{Cu}$ fibers. They found that the lithium metal is deposited on the tip of the previously formed lithium dendrites and promote the growth of the dendrites on the planar $\mathrm{Cu}$ foil. In contrast, numerous protuberant tips on the fibers in the skeleton of the $3 \mathrm{D} \mathrm{Cu}$ foil serve as the nucleation sites. The electric field is uniform and the charges are homogeneously distributed along the $\mathrm{Cu}$ skeleton, eventually leading to uniform lithium deposition. The freestanding $\mathrm{Cu}$ nanowire network also exhibited high Coulombic efficiency (98.6\% in average in 200 cycles) and outstanding rate performance owing to the suppressed lithium dendrite growth and the high conductivity of this network (Lu et al., 2016). In addition, Ma et al. (Ma et al., 2019) fabricated a 3D porous current collector composed of interconnected $\mathrm{Cu}$ nanoparticles by a facile one-step electro-deposition process. Stable cycling performance was obtained in cells with liquid and solid electrolytes.

In addition to the directly synthesizing $\mathrm{Cu}$ nanowires and nanoparticles, the 3D Cu current collectors can also be obtained by de-alloying the Cu-containing alloys (An et al., 2018; Zhang W. et al., 2019; Shi et al., 2019; Zhang D. et al., 2020). Shi et al. (Shi et al., 2019) constructed a 3D porous structure by chemically removing $\mathrm{Ga}$ from $\mathrm{CuGa}_{2}$, achieving a cycling life for over $700 \mathrm{~h}$ without formation of lithium dendrites. Similarly, a 3D Cu current collector was produced by chemically de-alloying a commercial bimetallic $\mathrm{Cu}-\mathrm{Zn}$ alloy tape (Yun et al., 2016). The continuous $\mathrm{Cu}$ skeleton acts as a current collector for fast electron transport while the interconnected pores provide a large internal surface area and room to accommodate the deposited lithium. However, it is difficult to precisely regulate the structure of the pores on the $3 \mathrm{D} \mathrm{Cu}$ current collector for a uniform porous structure during the chemical de-alloying process. Meanwhile, the $3 \mathrm{D} \mathrm{Cu}$ presents poor mechanical strength and low electrical conductivity due to its high porosity. Electrochemical etching was developed to solve this problem (Zhao et al., 2018). The 3D Cu consists of uniform pores and a compact framework can be obtained by electrochemically etching a $\mathrm{Cu}-\mathrm{Zn}$ alloy tape by the linear sweep voltammetry (Zhao et al., 2018).

Other materials were also applied as $3 \mathrm{D}$ porous current collectors, such as Ti (Zhang X. et al., 2019; Huang et al., 2019), Ni (Yu et al., 2018; Yue et al., 2018) and various carbon materials (Shen et al., 2018; Zuo et al., 2018; Liu F. et al., 2019). Lu et al.(Lu et al., 2017) designed a Cu@Ni coreshell nanowire network as the scaffold to accommodate the lithium plating/stripping inside to maintain the volume of the anode during battery cycling. In comparison with the metallic current collectors, the carbon-based current collectors are light, highly flexible and with large specific surface areas. The application of the porous graphite (Sun et al., 2016; Jin S. et al., 2017), carbon nanotubes (Shen et al., 2018; Zuo et al., 2018; Liu F. et al., 2019), porous graphene (Cheng et al., 2015; Zhang et al., 2016; Liu S. et al., 2018), graphitized carbon fibers (Liu et al., 2017; Yang et al., 2017; Zhang R. et al., 2018; Wang et al., 2019) have also been explored.

The advantages of the $3 \mathrm{D}$ porous current collectors used in lithium metal batteries include: 1) the high conductivity of the current collector materials and the interconnected 3D structure ensure the rapid charge transfer between the electrons and $\mathrm{Li}^{+}$ ions in the entire network. The uniform electric field and $\mathrm{Li}^{+}$ion distribution result in the uniform lithium deposition; 2) the large specific surface area can reduce the local current density and inhibit the growth of the lithium dendrites; 3 ) the pores of the $3 \mathrm{D}$ framework structure can accommodate the deposited lithium, reduce the volume change of the electrode during cycling, and increase the lithium plating capacity; and 4) the 3D structure can avoid stress concentration and bear the stress evenly owing to its excellent mechanical properties. However, there are also some problems to be solved such as 1) the high specific surface area leads to the abundant side reactions between the lithium metal and the electrolyte, which results in a considerable consumption of lithium and electrolyte; 2) the large volume of the 3D current collector results in low volumetric energy density of a full cell; and 3) more liquid electrolyte is needed for the $3 \mathrm{D}$ current collectors, which reduces the specific energy of a lithium metal cell. Therefore, a reasonable design of the $3 \mathrm{D}$ current collector including the porosity, surface area, and amount of lithium loading is critically important to the application of the metal lithium batteries.

\subsection{Selection of Current Collector Texture}

The texture of the current collector is another critical factor that affects the lithium deposition and its cycling. That is, lithiophilic materials are preferred as the current collectors. On one hand, the lithiophilic materials can reduce the nucleation barrier for the heterogeneous nucleation of lithium, which decreases the nucleation overpotential and thus increases the energy conversion efficiency. On the other hand, the interaction between the lithium ions and the lithiophilic materials enables a homogeneous distribution of the $\mathrm{Li}^{+}$ions, resulting in a uniform and smooth lithium deposition. These lithiophilic current collectors can be divided into two categories according to their reactivity with lithium, the electrochemically reactive substrates that can react with lithium ions during lithium plating, and the substrates with polar functional groups on the surface that can attract lithium ions.

\subsubsection{Electrochemically Reactive Substrates}

Prior to the lithium nucleation, the electrochemically active substrates react with the lithium ions by intercalation, alloying and/or conversion reactions. The lithium nuclei are subsequently formed on the reaction product, rather than on the substrate material directly. The reaction products can be $\mathrm{Li}_{\mathrm{x}} \mathrm{C}_{6}$ on the carbon materials (by intercalation reaction), Li-containing alloys on lithiophilic metals or nonmetals (by alloying reaction), and LiX on the metal compounds (by conversion reaction), respectively. These Li-containing products have strong affinity to lithium, thus reducing the lithium nucleation barriers. In addition, the situation is complicated in some lithiophilic metal substrates such as Ag and Mg. Lithium exists in the form of alloys rather than metallic lithium particles deposited on the alloy surface during lithium plating. The metallic lithium nuclei are not formed because lithium reacts with the substrates to form solid solution alloys. The lithium deposition/ dissolution behaviors on such metals will be discussed in Section 3 of this review. 


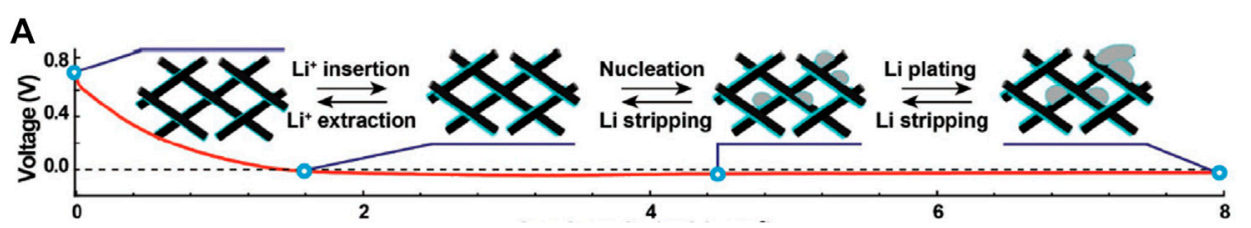

B

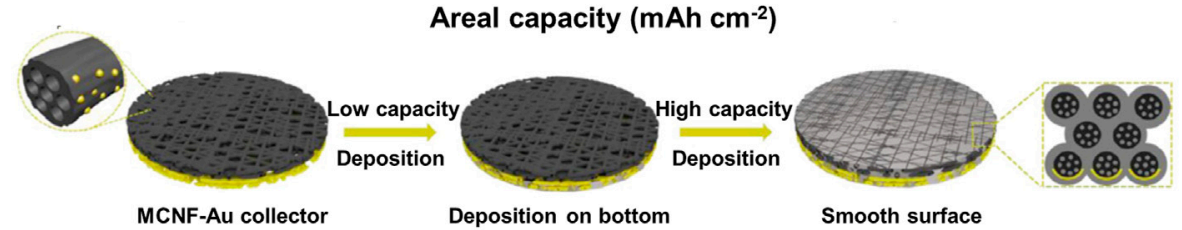

C

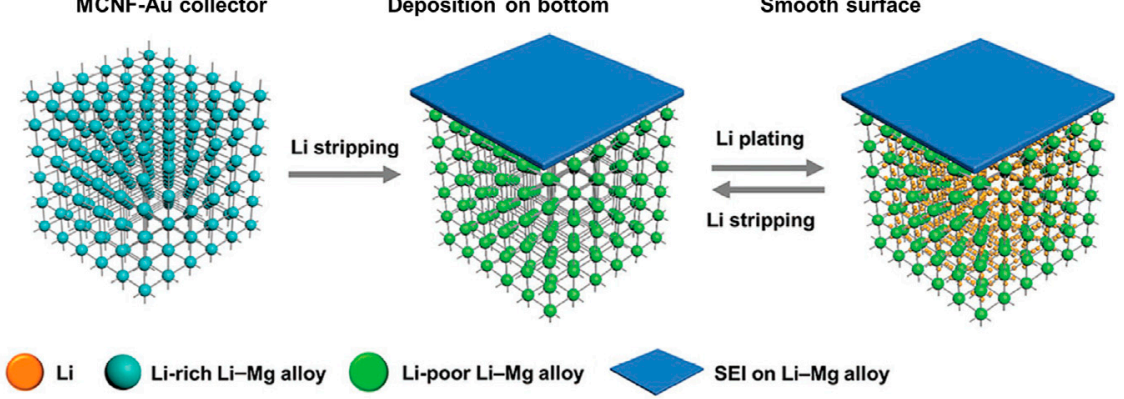

D

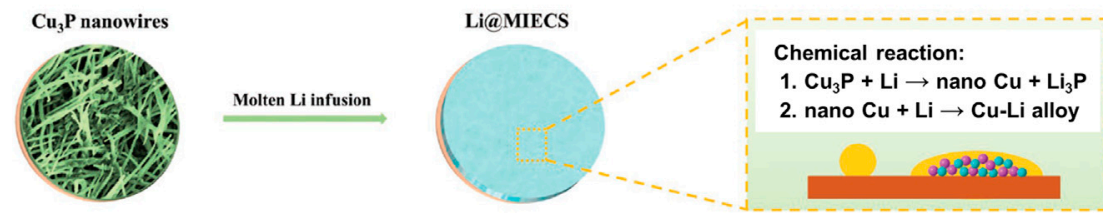

FIGURE 1 | Illustration of the discharge/charge process of the graphitized carbon fiber electrode (A). Reproduced from Zuo et al., 2017 with permission. Schematic of the Li depositing behaviors on the Au-modified multichannel carbon fiber paper (MCNF-Au) collector (B). Reproduced from Liao et al., 2020 with permission. Schematic structure in the bulk and on the surface for the Li-Mg alloy anodes during Li stripping/plating process (C). Reproduced from Kong et al., 2019 with permission. Schematic illustration of the preparation of the mixed ion/electron-conducting skeleton (MIECS). The possible chemical reaction between $\mathrm{Cu}_{3} \mathrm{P}$ and molten $\mathrm{Li}$ is used to construct a MIECS containing $\mathrm{Li}_{3} \mathrm{P}$ and Cu-Li alloy phase (D). Reproduced from Sun et al. (2019b) with permission.

Carbon materials are the most common current collector material for the lithium metal deposition, in which the lithium ions are generally stored by intercalation or adsorption before lithium nucleation. This pre-lithiation behavior enhances their affinity with the deposited lithium. Various carbon materials (few-layer graphite or multilayer graphene (Liu S. et al., 2018; Deng et al., 2018; Liu W. et al., 2019; Ren et al., 2019), carbon nanotubes (Wang Y. et al., 2017; Guo et al., 2018; Guo et al., 2019), carbon fiber (Lin et al., 2019; Zhang Y. et al., 2020; Liao et al., 2020; Kwon et al., 2021), etc.) have been proved effective for mitigating the Li dendrite growth due to their porous structure and/or affinity with lithium. Kwon et al. (Kwon et al., 2021) reported an atomically defective carbon current collector where multi-vacancy defects induce homogeneous SEI formation, uniform lithium nucleation and growth to obtain a dense lithium morphology. Zuo et al. (Zuo et al., 2017) developed graphitized carbon fiber electrode to enhance the Li storage capacity (Figure 1A). The as-obtained anode can deliver an areal capacity as high as $8.0 \mathrm{mAcm}^{-2}$ without obvious formation of lithium dendrites.

Apart from the carbon materials, numerous metals and nonmetals that can form alloys with lithium have been utilized in lithiophilic current collectors, including Ag (Xue et al., 2018; Zhang R. et al., 2018; Lee Y.-G. et al., 2020), Au (Yan et al., 2016; Liao et al., 2020; Corsi et al., 2021), Si (Zhao et al., 2017; Chen L. et al., 2019; Yue et al., 2021), Zn (Jiang et al., 2020; Zheng et al., 2020), Al (Kim et al., 2019; Ye et al., 2019a), Mg (Xu Q. et al., 2020; Gao et al., 2021) and B (Liu Q. et al., 2018; Wu et al., 2020). They can reduce the lithium nucleation overpotential and improve the lithium cycling stability. These materials are used as coatings and 3D lithiophilic hosts. In some studies, several (non)metals are coated on the current collectors, in the form of thin films or nanoparticles (Peng et al., 2019; Ye et al., 2019a). We coated silver nanoparticles on the carbon fiber paper to guide the uniform lithium plating, effectively reducing the nucleation overpotential and ensuring the stable electrochemical cycling (Li X. et al., 2019). A multichannel carbon fiber paper with $\mathrm{Au}$ modification on one side was developed as the collector (Liao et al., 2020). The special multichannel geometry generates structural stress that pushes the $\mathrm{Li}^{+}$ions to deposit into the inner channels; the Au modification on the back side of the matrix enables lithium to fill the deep voids between the fibers via heterogeneous nucleation, guaranteeing the maximum utilization of the possible spaces (Figure 1B). Most of the 

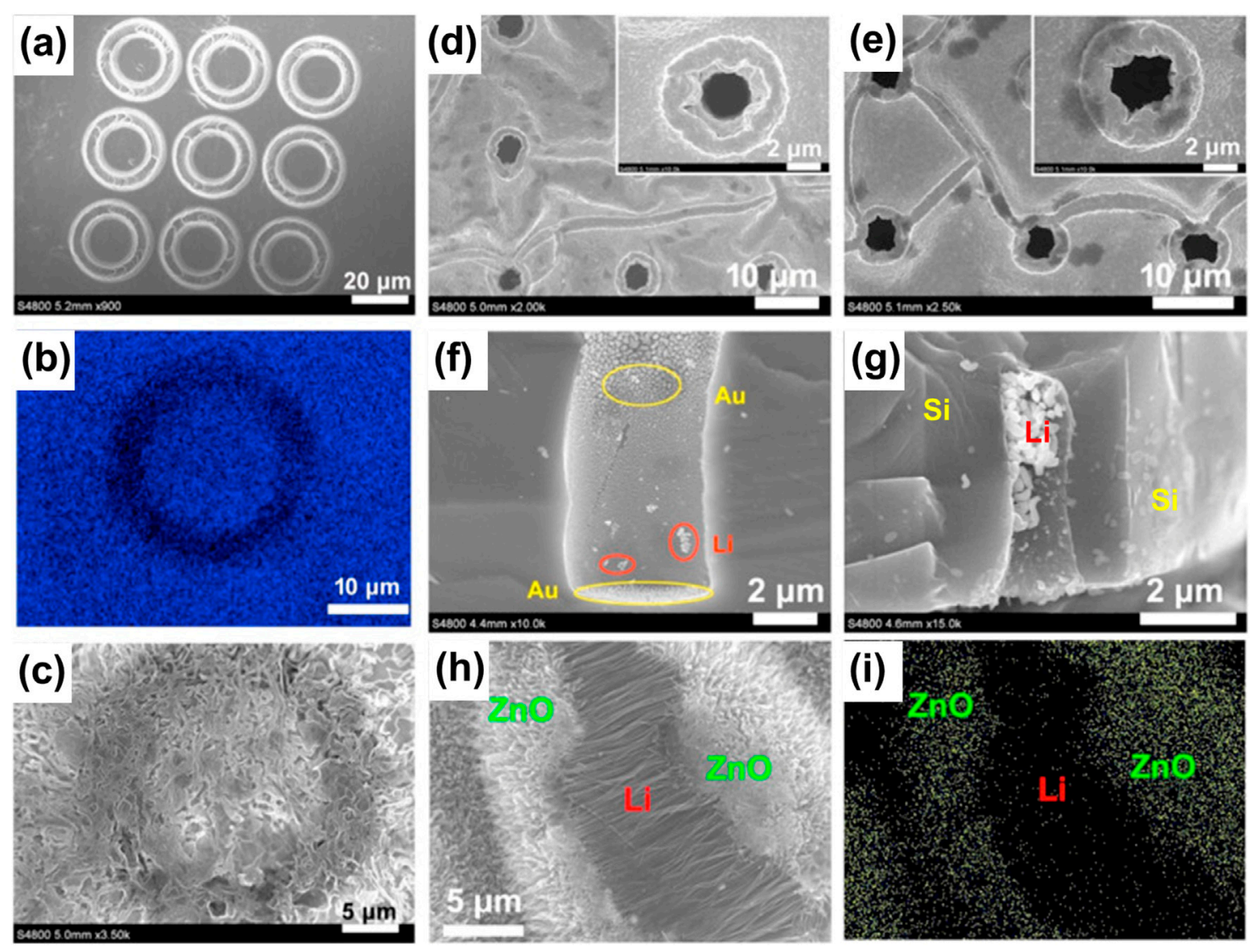

FIGURE 2 |SEM image of the as-prepared FIB-etched patterns on Ti foil (A), the corresponding Ti-EDS mapping (B) and SEM image after lithium deposition (C). Lideposited Si pattern with different lithium plating capacities (D and $\mathbf{F}$ and $\mathbf{E}$ and $\mathbf{G})$. SEM image of the Li nanowires array grown between the ZnO arrays (H), and the corresponding EDS mapping (I). Reproduced from Li et al. (2017).

studies on the lithiophilic surface coating were focused on Ag and $\mathrm{Au}$, but these expensive metals cannot be applied in large scale on the current collectors. Therefore, more efforts are required to develop economical metals as the surface coating on the current collectors. In other cases, some (non)metals are also used as 3D lithiophilic hosts to accommodate the deposited lithium, reduce the local current density, and suppress the formation of the lithium dendrites (Kong et al., 2019; Pathak et al., 2020; Ye et al., 2020; Zhuang et al., 2020). The 3D substrate functioned with the lithiophilic Li-Zn alloy surface was designed to induce the uniform lithium deposition along the conductive skeleton, effectively suppress the dendrite growth and volume expansion (Ye et al., 2020). Kong et al.(Kong et al., 2019) investigated a Lirich Li-Mg alloy as an anode for the Li-S batteries (Figure 1C). The mixed electron and Li-ion conducting matrix of the Li-leach $\mathrm{Li}-\mathrm{Mg}$ alloy as a porous skeleton structure can also be formed after delithiation, which can ensure the structural integrity of the anode in the bulk during Li stripping/plating process. It should be noted that, in comparison with the surface coating, more lithiophilic materials are required to construct the 3D current collectors. Cautious should be taken about the amount of the lithiophilic material in the $3 \mathrm{D}$ current collectors in order to ensure the high practical gravimetric and volumetric energy density of the anode.

Some metal compounds that undergo conversion reactions with lithium can also affect the morphology of the deposited lithium and reduce the nucleation overpotential, such as metal oxides (ZnO (Jin C. et al., 2017; Sun et al., 2019a; Zhao et al., 2019; Yue et al., 2020), $\mathrm{CuO}$ (Zhang C. et al., 2018; Huang et al., 2019; Wei et al., 2020; Huang et al., 2021), $\mathrm{Cu}_{2} \mathrm{O}$ (Zhang Q. et al., 2018) $\mathrm{SnO}_{2}$ ), fluorides $\left(\mathrm{CuF}_{2}\right.$ (Yan C. et al., 2018), $\mathrm{NiF}_{2}$ (Peng et al., 2017)), phosphides $\left(\mathrm{Cu}_{3} \mathrm{P}\right)$ (Zhang C. et al., 2019; Sun et al., 2019b) and nitrides $\left(\mathrm{Cu}_{3} \mathrm{~N}\right)$ (Lee D. et al., 2020; Li et al., 2021). Zhang et al. (Zhang C. et al., 2018) prepared lithiophilic $\mathrm{CuO}$ nanosheets modified $\mathrm{Cu}$ foil by a simple wet chemical reaction to stabilize the lithium nucleation and alleviate the growth of lithium dendrites, thus enhancing the lithium plating and stripping performance. The electrochemically lithiated $\mathrm{Cu}_{3} \mathrm{P}$ nanowires was demonstrated to induce the dendrite-free lithium growth as well as enhance Coulombic efficiency (Sun et al., 2019b) (Figure 1D). It is key to control the thickness and surface state of the compound layer to achieve the best performance because the introduction of metal compounds generally results in poor electronic conductivity. 

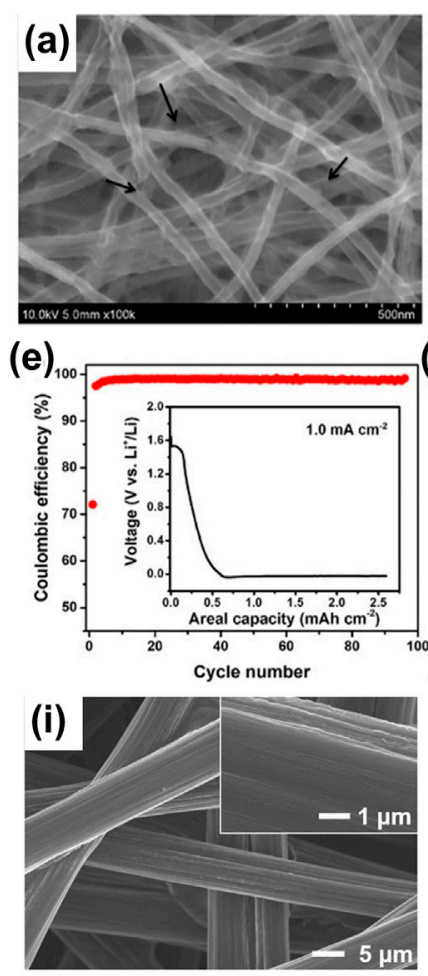
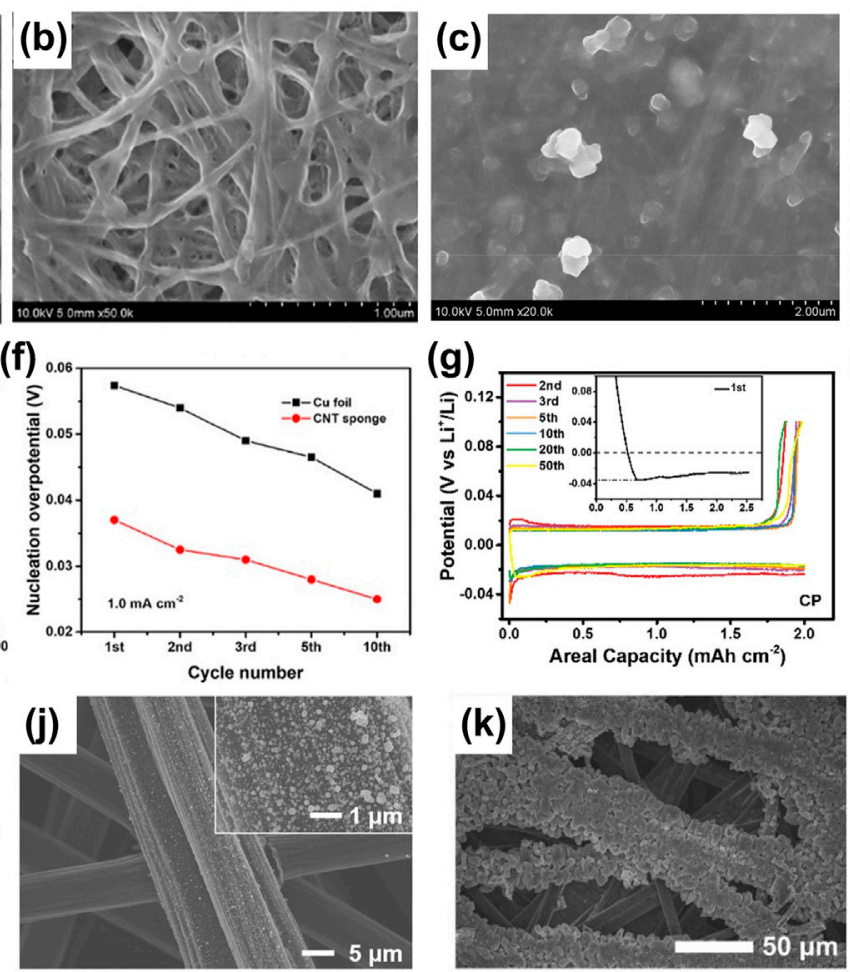

(g)
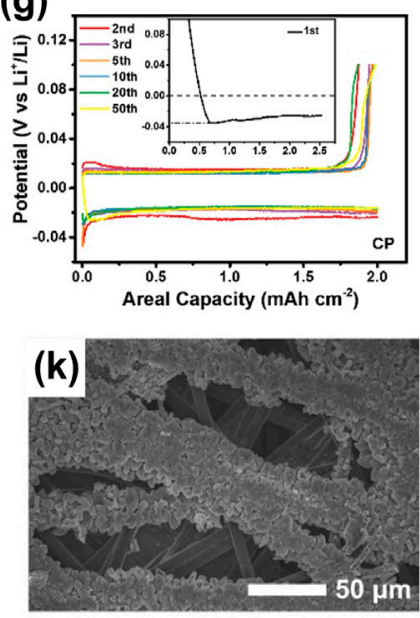

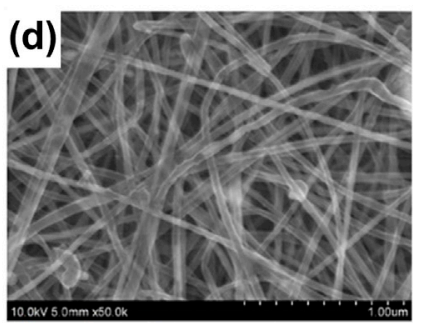

(h)
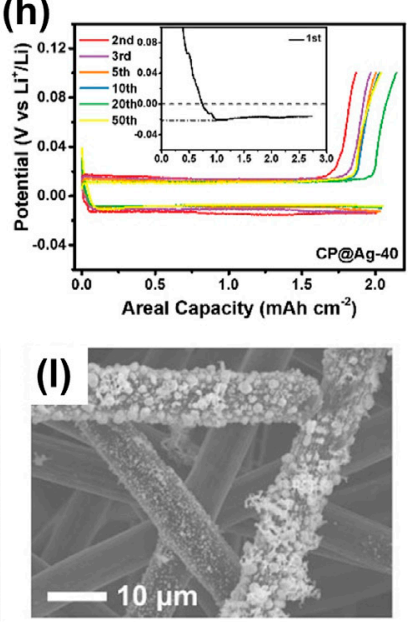

FIGURE 3 | Morphology of the CNT sponge at different stages of lithium plating and stripping: lithium plating $0.04 \mathrm{~mA} \mathrm{~h} \mathrm{~cm}^{-2}$ (A), lithium plating $2.0 \mathrm{~mA} \mathrm{~h} \mathrm{~cm}^{-2}$ (B), lithium plating $10.0 \mathrm{~mA} \mathrm{~h} \mathrm{~cm} \mathbf{~}^{-2} \mathbf{( C )}$ and lithium stripping after 20 cycles (D). The electrochemical performances of the cells with CNT sponge as the substrate (E). Comparison of the evolution of the nucleation overpotential on the CNT sponge and on Cu foil with cycling (F). Reproduced from Yang et al. (2019b) Comparison of the electrochemical performances of the cells with CP (G) and CP@Ag $\mathbf{( H )}$ as the lithium deposition substrates. The evolution of morphology of the CP@Ag during lithium plating/stripping: the commercial CP (I), the as-prepared CP@Ag (J), lithium plating 2.0 mA h cm ${ }^{-2}$ on CP@Ag (K), lithium stripping after 10 cycles (L). Reproduced from Li et al. (2019b).

\subsubsection{Substrates With Polar Functional Groups on Surface}

Some polar functional groups can enhance the uniform nucleation of lithium, reduce the effective current density, and homogenize the $\mathrm{Li}^{+}$flux to inhibit dendrites. The binding energy of the lithium ions with these polar functional groups is larger than that with the copper or lithium atoms (Chen X. et al., 2019). Therefore, the polar functional groups can adsorb a considerable amount of the $\mathrm{Li}^{+}$ions to overcome the electrostatic interactions between the $\mathrm{Li}^{+}$ions and the protrusions, avoiding the aggregation of the $\mathrm{Li}^{+}$ions around the protuberances. Zhang et al. (Cheng et al., 2016) employed glass fibers containing plenty of polar functional groups as an interlayer between the lithium metal anode and separator. The chemical force from the polar functional groups hinders the diffusion of lithium ions to the lithium metal protuberances and reduces the lithium deposition in the vertical direction. Kong et al. (Kong et al., 2018) proposed a nitrogen-doped graphene structure with abundant polar functional groups, including pyrrolic, pyridinic, and quaternary nitrogen atoms. They revealed that the $\mathrm{N}$-doped carbon can significantly reduce the Li nucleation overpotential and thus render a uniform Li deposition at the lithiophilic sites (Chen X. et al., 2019). In addition, the-NH (Niu et al., 2019) and $-\mathrm{C}=\mathrm{O}$ (Lin et al., 2016) functional groups also exhibit large binding energies to lithium. Using the polar functional groups to modify the surface is effective in improving the lithiophilicity of the current collectors, but it decreases the electronic conductivity and thus, the amount of these polar functional groups added to the surface needs to be properly controlled.

The lithiophilic materials can reduce the nucleation barrier and achieve a dendrite-free lithium deposition. However, some efforts should be taken to the following issues in the application of the lithiophilic current collectors. 1) Au and Ag substrates have been extensively studied, but their high cost will limit their largescale applications. Some cheap metals that form alloys with lithium need to be developed as the alternatives; 2) additional attention should be paid to the stability of the alloy materials during cycling. They may undergo large volume change and phase transitions during the repeated alloying/dealloying, leading to the structural collapse or the particle fracture; and 3) the introduction of lithiophilic materials such as lithiophilic metal oxides and polar functional groups may cause poor electronic conductivity. Therefore, the cost, amount and structural changes of the lithiophilic materials need to be considered on designing the lithiophilic current collectors in order to meet the multiple requirements of the lithium metal batteries. 


\section{SOME OF OUR WORK}

\subsection{Selective Deposition on Micro-nano Patterns}

Many studies have demonstrated the feasibility of changing the lithium plating/stripping behaviors by modifying the physical and chemical properties of the electrode surface (Zhang Y. et al., 2017; Wang A. et al., 2018; Zou et al., 2018; Zhai et al., 2020). However, the fundamental understanding on how the electrode surface morphology affects the deposition behavior is inadequate and more effort is required to clarify the growth mechanism of the lithium dendrites. We found that the deposition of lithium metal can be guided to the micro-patterns on a Ti foil (Li et al., 2017). With this phenomenon as a start, we reported the preferential deposition of lithium metal in micropores with different physical and chemical properties such as the wells on $\mathrm{Si}$ wafer and the arrays of $\mathrm{ZnO}$ nanowires. We proposed the possible mechanism of preferential lithium deposition in pits and extended these findings in the narrowly defined pits into the generalized pores composed of nanostructures, which can achieve higher-capacity lithium metal deposition to meet the practical requirements of the lithium metal batteries.

In the first place, the Ti foil substrate with micro-grooves was employed for lithium deposition. By the energy dispersive spectroscopy (EDS) and scanning electron microscopy (SEM) observation, the micro-patterns on the foil was proved to be the location where the lithium metal prefers to deposit (Figures $2 \mathrm{~A}-\mathrm{C}$ ). These provide solid evidence for the guiding effects of the micro-pore on the deposition of the lithium metal.

On the basis of this, more micro- and nano-architectured electrodes including porous $\mathrm{Si}$ and $\mathrm{ZnO}$ electrodes, were prepared to control the lithium deposition. It was found that lithium is deposited at first on the bottom of the well (Figures 2D,F) and then grows in it (Figures 2E,G) on the porous Si electrode, which suggests that the lithium deposition is well confined within the wells. This confinement can reduce the contact area between lithium metal and the electrolyte during cycling and suppress the side reactions between them, and thus contributed to a much higher Coulombic efficiency for the cell with the patterned $\mathrm{Si}$ wafer electrode than the planar Si wafer counterpart.

Apart from the two cases of grooves on Ti foil and wells on $\mathrm{Si}$ wafer, the pit sizes of which are limited to a few microns for the sake of fabrication efficiency and cost, the arrays of the $\mathrm{ZnO}$ nanowires, with gaps or crevices between the oriented nanowires, is regarded as natural nano-pores. The electrodes with the arrays of $\mathrm{ZnO}$ nanowires were prepared, and the results after lithium plating show the presence of a new lithium array (or bundle) of oriented nanowires between the $\mathrm{ZnO}$ arrays, the shape of which is like one between two pieces of jigsaw puzzle (Figures 2H,I), confirming the controlled growth of lithium within the crack of the $\mathrm{ZnO}$ arrays.

We proposed a mechanism to explain the preferential lithium deposition in pits. The lithium metal and the electrolyte are regarded as an electron conductor and $\mathrm{a}^{+}{ }^{+}$conductor, respectively. The concave pits on the metal electrode are opposite to the tip protrusions of the electrolyte, between the pit and the tip is the electrode/electrolyte interface. Clearly, the tip of the electrolyte is the point at which the $\mathrm{Li}^{+}$ions are gathered and the electric current is the strongest, on the basis of the classical electromagnetism principles. Therefore, it is understandable that most of the $\mathrm{Li}^{+}$ions can receive electrons from the metal electrode and preferentially deposit in the pit.

This study revealed that the electrochemical activity of the substrate materials, in comparison with the well-designed surface structures, plays a less important role in determining where the lithium be deposited. Therefore, it is viable to realize controlled lithium deposition by specifically designing the geometrical structure of the current collector.

\subsection{Lithiophilic (non)metals Induced Lithium Deposition}

The materials that can be electrochemically alloyed with lithium exhibit a certain degree of lithiophilicity, including nonmetals such as $\mathrm{C}$ and $\mathrm{Si}$, and metals such as $\mathrm{Ag}$ and $\mathrm{Au}$ (Yan et al., 2016). These materials can be used as current collectors to induce lithium deposition and reduce the lithium nucleation barrier. This group utilized CNT sponge and Ag-plated carbon fiber paper (CP@Ag) as the current collectors to achieve dendrite-free lithium deposition, which proves the feasibility of the alloying strategy in improving the performance of the secondary lithium batteries. Afterwards, we conducted systematic research on the lithium plating/stripping behaviors on a variety of lithiophilic materials, clarified the relationship between the structural variation of the alloys and the lithium plating/stripping behavior, and proposed a principle for the rational selection of the lithium deposition substrates.

\subsubsection{Lithium Plating on CNT Sponge}

With years of development, many strategies of modification have been tried but problems remain unsolved such as the lithium dendrites growth and the low Coulombic efficiency. Noticing the correlation between the porous current collector and the lithium deposition and on the basis of our understandings for the lithium deposition on the lithiophilic materials, we firstly proposed to use the CNT sponge (CNTs) as a porous lithiophilic current collector to improve the lithium deposition and electrochemical performance (Yang et al., 2019b). The commercial CNTs, with its commercial availability and high specific surface area, especially its graphitic-amorphous carbon composite feature, was utilized as a 3D current collector to guide the dendrite-free lithium deposition. SEM images and the nitrogen adsorption-desorption isotherm exhibit the 3D porous network of the CNT sponge and the porous structure of each carbon nanotube, respectively. The Raman spectrum demonstrates the graphiticamorphous carbon composite structure of the CNT sponge.

The use of CNTs as a 3D current collector improves the electrochemical performance of LMB. With a current density of $1.0 \mathrm{~mA} \mathrm{~cm}$ and a lithium deposition capacity of $2.0 \mathrm{~mA} \mathrm{~h} \mathrm{~cm}{ }^{-2}$, the Coulombic efficiency of the Li|CNT cell is $72.1 \%$ in the first cycle, but sharply increases to 97.5 and $98.8 \%$ in the second and 10th cycles, respectively. It then maintains above $98.5 \%$ in the subsequent 90 cycles. (Figure 3E). 
The lithium plating/stripping electrochemical behaviors are quite different on CNTs and on bare $\mathrm{Cu}$. On one hand, the lithium nucleation overpotential on CNTs is much smaller than on bare $\mathrm{Cu}$ (Figure 3F), originated from the distinct surface properties of the two current collectors. A large amount of lithium is absorbed and condensed lithium is formed on the CNT sponge due to its microporous characteristics, leading to a reversible lithium storage capacity of $150 \mathrm{~mA} \mathrm{~h} \mathrm{~g}^{-1}$ above $0.0 \mathrm{~V}$ on the CNTs. Therefore, the pre-lithiated CNTs has a higher chemical affinity with lithium metal, reducing the nucleation overpotential. On the other hand, the Li||CNT sponge cell can run stably for $400 \mathrm{~h}$, owing to the structural stability of CNT ensured with the graphitic-amorphous carbon composite structure. The co-intercalation of the solvated lithium ions and the exfoliation of the layer-structured graphite are inevitable during the prelithiation of the graphitic carbon in the ether-based electrolytes. Therefore, such composite structure is important for the structural stability of CNTs.

The morphology evolution at different lithium plating capacities shows that the lithium is deposited uniformly on the skeleton of the CNTs at first and then gradually fills the voids in the sponge network (Figures 3A,B). No lithium dendrites are observed even if the lithium plating areal capacity is as high as $10.0 \mathrm{~mA} \mathrm{~h} \mathrm{~cm}^{-2}$ (Figure 3C). After lithium stripping, both the $3 \mathrm{D}$ skeleton of the sponge and its graphite-amorphous carbon composite structure are well preserved (Figure 3D). The dendrite-free lithium deposition is attributed to the high specific surface area, which ensures a good electrical contact between the electrode and the electrolyte and increases the density of the lithium nucleation sites, thereby decreasing the local current density on the CNTs.

\subsubsection{Improved Lithium Deposition on Silver-Plated Carbon Fiber Paper}

Although the pre-lithiation above $0.0 \mathrm{~V}$ enhanced the affinity of the carbon materials to lithium, the nucleation barriers for lithium plating on the carbon materials are still large (for example, the nucleation overpotential is $37 \mathrm{mV}$ on the CNTs in the first cycle) (Yang et al., 2019b), which hinders the uniform and controlled lithium deposition. It was reported that there are no nucleation barriers on the metals that exhibit some solubility to lithium, such as Ag and Mg (Yan et al., 2016). Therefore, the application of these metals as lithiophilic seeds can further reduce the nucleation barrier and control the lithium deposition on the carbon substrates.

We pre-plated nano-sized silver particles on commercial carbon fiber paper $(\mathrm{CP})$ via the silver mirror reaction and used the silver-coated CP (CP@Ag) as a porous current collector for lithium deposition (Li et al., 2019b). The diameter of most of the silver particles is a few tens of nanometers (Figures 3I,J). X-ray diffraction (XRD) and X-ray photoelectron spectroscopy (XPS) demonstrate the existence of the metallic silver.

The presence of the silver nanoparticles improves the lithium plating/stripping performance on the carbon fiber paper. The lithiophilic silver particles alloy with the lithium and guide the subsequent lithium plating on the carbon fibers (Figure 3K), effectively reducing the polarization and improving the energy conversion efficiency of the lithium anode. The nucleation and deposition overpotentials decrease from 35 to $26 \mathrm{mV}$ on CP to 21 and $18 \mathrm{mV}$ on $\mathrm{CP} @ \mathrm{Ag}$ in the first cycle, respectively (Figures 3G,H). The CP@Ag cell exhibits stable cyclability at a current density of $0.5 \mathrm{~mA} \mathrm{~cm}^{-2}$ for about $1,000 \mathrm{~h}$.

The electrochemical impedance spectroscopy (EIS) indicates that the SEI film resistance and the charge transfer resistance are smaller on the CP@Ag than on the naked CP. This illustrates that the SEI film with higher ion conductivity is formed on the CP@Ag. It is easier for the lithium ions to be reduced to metallic lithium on the CP@Ag. These explain the decrease of the nucleation and the deposition overpotentials.

The evolution of the morphology during lithium plating indicates that the silver particles can guide the uniform deposition of lithium on the carbon fibers. The Ag particles still adhere tightly to the carbon fiber after 10 cycles (Figure 3L). The strong adhesion of this chemically plated silver ensures the cycle stability and high Coulombic efficiency of the lithium plating/stripping. Other lithiophilic metals can also induce uniform lithium deposition, and some cheap metals that form alloys with lithium can be good alternatives to silver, such as Zn, Al (Ye et al., 2019a) and Si (Tang et al., 2018).

\subsubsection{Phase Diagram Determined Lithium Plating/ Stripping Behaviors}

On the basis of the studies on CNT, CP@Ag and a number of other lithiophilic materials as current collectors, it can be found that the lithium plating/stripping behaviors are closely dependent on the substrate material. Significant differences have been observed in the nucleation overpotential, morphology of the deposited lithium (or alloy) and their cycling performances when different materials are used as the substrates. However, the fundamentals for these differences remain unclear and some understandings are even problematic. It is essential to clarify the reason for the different lithium plating/stripping behaviors and find the principles for the rational selection and design of the substrate materials.

With the phase transition process of a series of lithiophilic materials and density functional theory (DFT) calculations, we clarified that the lithium plating/stripping behaviors on the (non) metal substrates are basically determined with the binary phase diagrams of the lithiophilic materials (Zhang et al., 2021b). The lithium plating/stripping process on the $\mathrm{Ag@Cu}$ substrate is always accompanied with the phase transition of the $\mathrm{Li}-\mathrm{Ag}$ alloys. During lithium plating on the Ag@Cu substrate, the $\alpha$ phase Ag-rich solid solution, $\beta$ phase (LiAg), $\gamma$ phase Li-Ag solution $\left(\gamma_{3}, \gamma_{2}, \gamma_{1}\right.$ phases $)$ and $\delta$-phase Li-rich solid solution appear in sequence (Figure 4A). A reversed process occurs as lithium is dissolved from the $\mathrm{Li}-\mathrm{Ag}$ alloys. The lithium exists in the form of Li-Ag solid solutions instead of free metallic lithium in these processes, because there are a series of solid solutions but no Li-Ag intermetallic compound in the Li-Ag phase diagram (Figure 4C). The solid-solution reaction permits the formation of the $\mathrm{Li}-\mathrm{Ag}$ alloys with any possible $\mathrm{Li} / \mathrm{Ag}$ proportion. Therefore, the phase transition runs through the entire Li-Ag phase diagram 
(a)

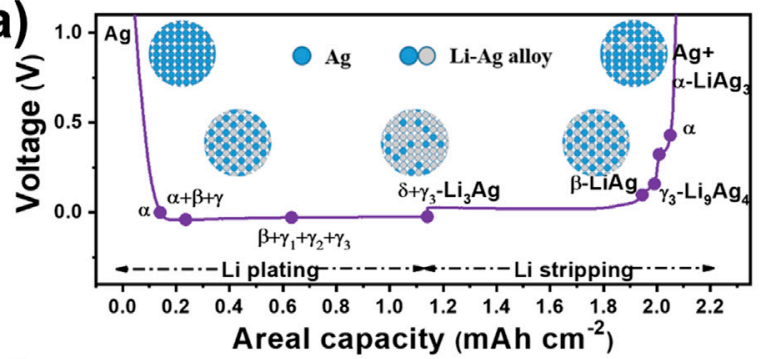

(c)

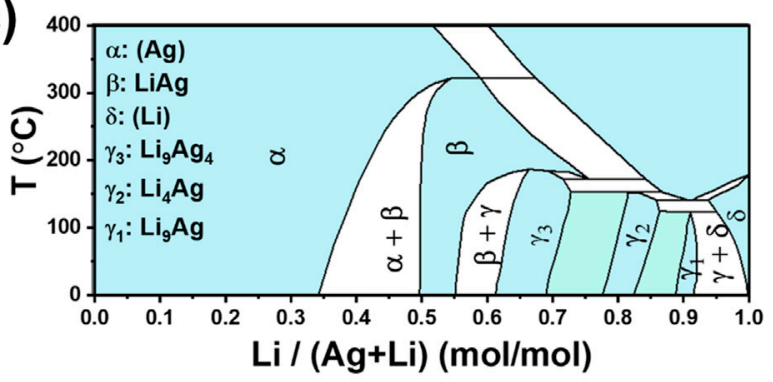

(b)

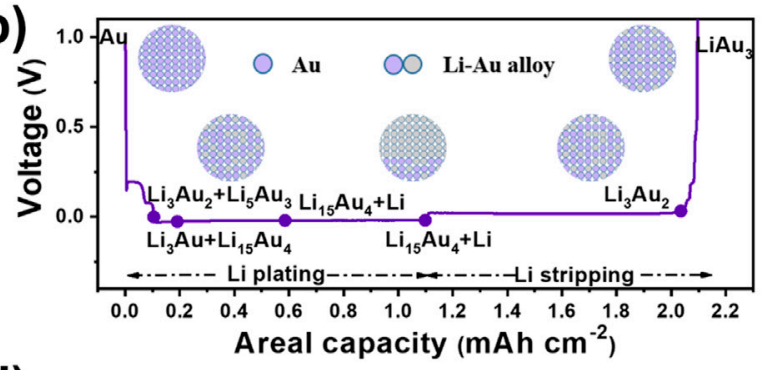

(d)

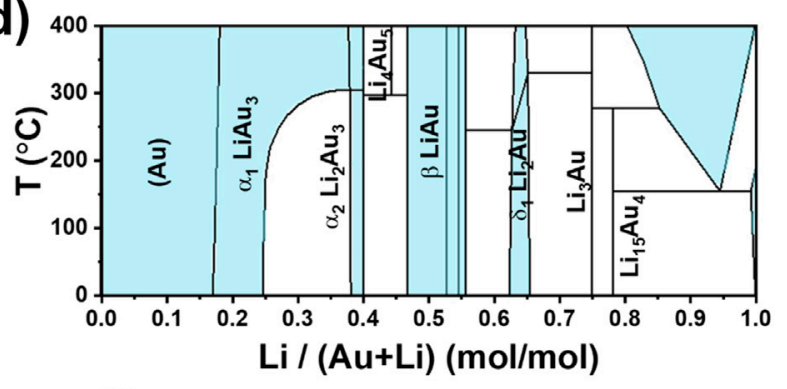

(e)
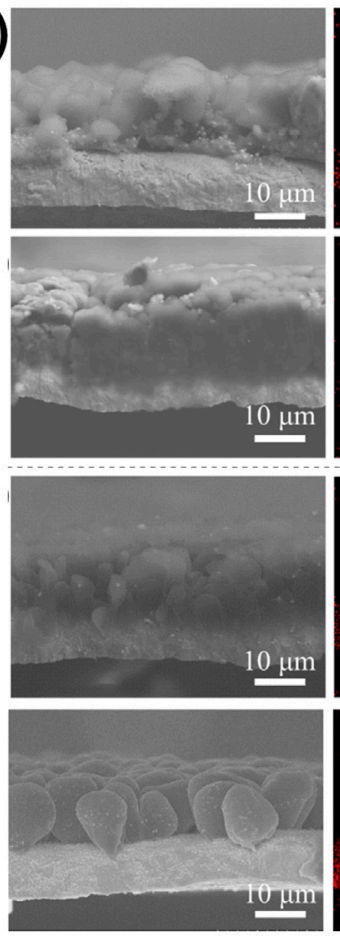
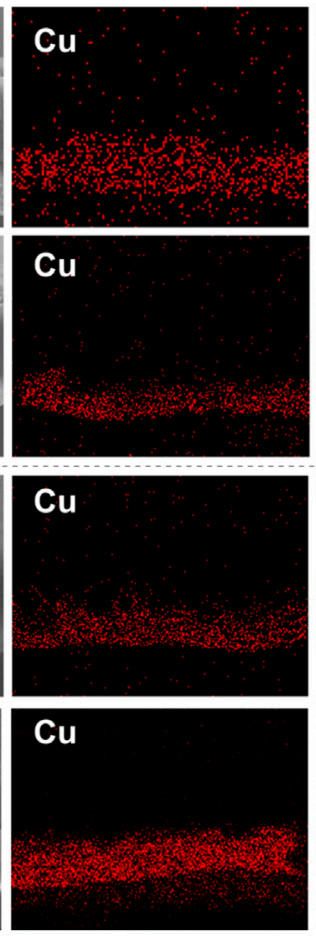
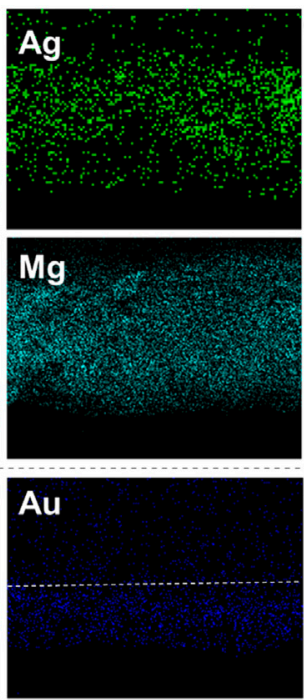

$\mathbf{Z n}$

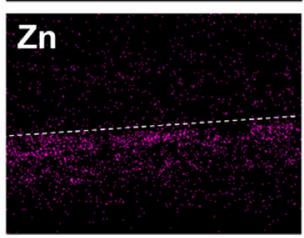

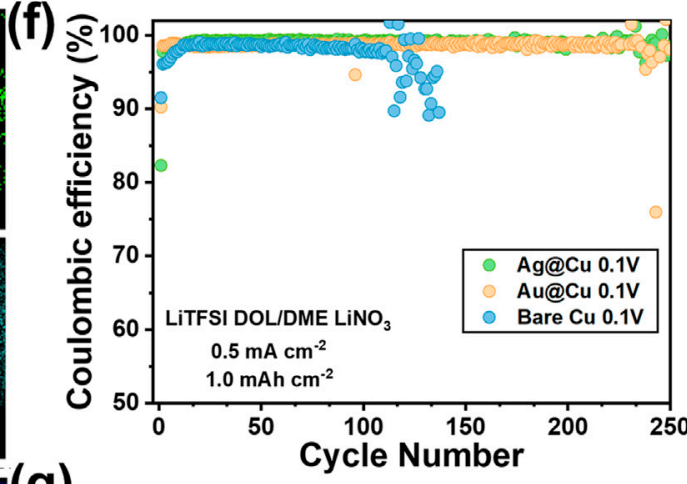

(g)

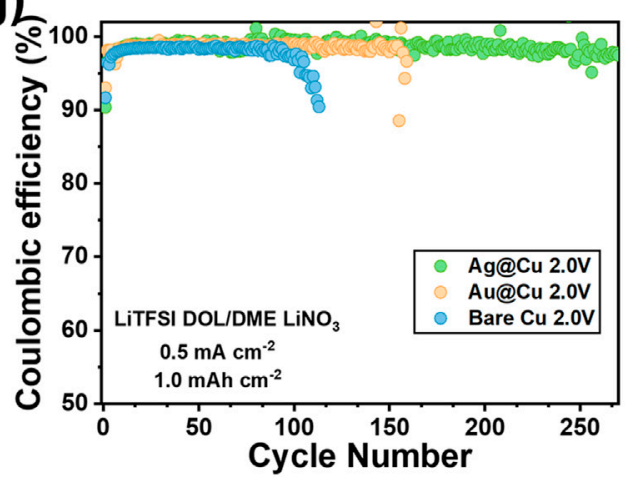

FIGURE 4 | Schematic illustration of the phase transition process on the Ag@Cu (A) and Au@Cu (B) substrates. The Li-Ag (C) and Li-Au (D) binary phase diagrams. SEM images of the cross section and the corresponding SEM-EDS mapping of Ag, Mg, Au, and $\mathrm{Zn}$ on Cu after lithium plating of $2.0 \mathrm{~mA}$ h $\mathrm{cm}^{-2}$ (E). Comparison of the Coulombic efficiency of the Lil|Cu cells using Ag@Cu, Au@Cu and Cu substrates and charge cutoff potentials of 0.1 V (F) and 2.0 V (G). Reproduced from Zhang et al. (2021b).

and mainly involves some single-phase transitions (solid solution reactions).

During lithium plating on the $\mathrm{Au} @ \mathrm{Cu}$ substrate, Au can only accommodate a small amount of lithium (the highest lithium content is $\mathrm{Li}_{15} \mathrm{Au}_{4}$ ) and the later deposited lithium exists as free lithium on the Au@Cu substrate (Figure 4B). The free lithium is firstly stripped, and then $\mathrm{Li}_{15} \mathrm{Au}_{4}$ is finally transformed to $\mathrm{LiAu}_{3}$ phase during lithium stripping. Therefore, the lithiation of $\mathrm{Au}$ reaches saturation faster than that of $\mathrm{Ag}$, followed by the deposition of free lithium. The existence of a series of intermetallic compounds in the $\mathrm{Li}-\mathrm{Au}$ phase diagram is the reason for such phase transition process (Figure 4D). There 
are many two-phase regions in the $\mathrm{Li}$-Au phase diagram, leading to the frequent two-phase transition and significant structural change.

The impact of the kinetic factors on the alloying/de-alloying behavior can be excluded by comparing the diffusion energy barriers of the metals in the $\mathrm{Li}_{3} \mathrm{Au}$ and $\mathrm{Li}_{3} \mathrm{Ag}$. The DFT calculations show that the diffusion energy barriers of $\mathrm{Ag}$ and $\mathrm{Au}$ atoms are large in the two alloys while those of lithium are very small and are similar in these two alloys. These suggest that the lithium diffusion plays a dominant role in the formation and phase transition of the alloys. It is the thermodynamic factor (the phase diagram), rather than the kinetic factors (the high diffusion coefficient) that determines the alloying behavior of lithium on the lithiophilic substrate (forming solid solutions or intermetallic compounds).

In combination with the binary phase diagrams, the lithiophilic (non)metals can be classified into two categories. The metals that only form solid solutions with lithium such as $\mathrm{Ag}$ and $\mathrm{Mg}$ can continuously accept the injected lithium to form solid solutions. In contrast, the (non)metals that intermetallic compounds are present in the phase diagrams can only accommodate a limited amount of lithium, such as $\mathrm{Au}, \mathrm{Al}, \mathrm{Zn}, \mathrm{Si}$ and $\mathrm{Sn}$. Free lithium is plated on top of the Li-saturated alloys and the latter cannot affect the lithium deposition anymore.

The SEM-EDS images show that $\mathrm{Ag}$ (or $\mathrm{Mg}$ ) is uniformly distributed in the direction perpendicular to the substrates, indicating the formation of $\mathrm{Li}-\mathrm{Ag}$ (or $\mathrm{Li}-\mathrm{Mg}$ ) solid solutions (Figure 4E). The scanning transmission electron microscopy (STEM)-EDS demonstrates that the alloy particles remain integrated though the $\mathrm{Li}$ concentration in the $\mathrm{Li}-\mathrm{Ag}$ alloy particles decreases from the surface to the internal. The Ag@ $\mathrm{Cu}$ substrate remains integrated after de-alloying, ensuring the stable lithium plating/stripping in the subsequent cycles. However, $\mathrm{Au}$ ( or $\mathrm{Zn}$ ) is only observed on the surface near the $\mathrm{Cu}$ foil (Figure 4E). Free lithium metal lies between the $\mathrm{Li}_{15} \mathrm{Au}_{4}$ alloy and the SEI layer.

The phase diagram characteristics strongly affect the lithium cycling behaviors on the lithiophilic substrates, embracing the morphology of lithium or alloys, the structural stability and the formation of the SEI layers. The $\mathrm{Li}-\mathrm{Ag}$ alloys guide the deposition of the large lithium particles at any lithium plating capacity. Porous particles are formed after lithium stripping, which act as the lithiophilic seeds to guide the condensed lithium deposition in the subsequent cycles. Different from that on $\mathrm{Ag@Cu}$, the $\mathrm{Li}-\mathrm{Au}$ alloy can no longer regulate the morphology of the deposited lithium after being covered with free lithium, resulting in formation of smaller deposited lithium particles. The deposited lithium on the Au@Cu substrate is looser than on the Ag@Cu substrate after long-term cycling, because the accumulated internal strains during the two-phase transitions promote the crumbling and dropping of $\mathrm{Au}$ from the $\mathrm{Au} @ \mathrm{Cu}$ substrate. A stable SEI film rich of fluorine is formed on the surface of the $\mathrm{Li}$-Ag alloys, and the porous Li-Ag alloy particles can support the SEI film to keep its integrity. However, many cycles are required and a lot of lithium is consumed before a protective SEI layer is formed on the Au@Cu substrate.
The phase diagrams determine the electrochemical behaviors of lithium deposition on different substrate materials. The lithium deposition on the material that only forms lithium solid solution shows stable nucleation overpotential, high Coulombic efficiency, long lifespan and high structural stability after deep de-alloying (Figures 4F,G). The excellent electrochemical performance is due to the slight structural changes, reduced side reactions and decreased dead lithium. The significant increase in the nucleation overpotential on the Au@Cu substrate after 50 cycles proves the fall-off of the Au particles caused by the large structure change of the Li-Au alloys.

The universality of this rule has been proved on a variety of metal substrates in the lithium metal batteries. This finding makes it possible to predict the electrochemical performance of alloy materials under different conditions and design alloy materials through alloy phase diagrams in secondary metal batteries.

\subsection{Lithium Deposition in CNT Cavity}

With a cavity inside, CNT is a native porous material. It was once expected in history to be an ideal container of lithium. However, all the efforts to fill the cavities with lithium failed. For the first time, we proposed a novel approach to realizing the lithium storage in the CNT cavities with the assistance of the transition metal carbides, such as iron carbide, inside the CNT to induce the lithium ions (Yang et al., 2021a).

The above CNT sponge was used as the substrate for lithium deposition, in the cavities of which is confirmed the presence of $\mathrm{Fe}_{3} \mathrm{C}$ and $\mathrm{Fe}_{2} \mathrm{C}\left(\mathrm{Fe}_{\mathrm{x}} \mathrm{C}\right)$ by the transmission electron microscopy (TEM). After the CNT sponge is discharged to $0.0 \mathrm{~V}$, crystalline metallic lithium is observed in the cavities accompanied with the iron carbides, as is proved by the cryogenic-TEM (cryo-TEM) and electron energy loss spectroscopy (EELS) (Figure 5A-C).

Another interesting finding is the location of the lithium metal-it is stored between the inner wall of the CNT and the iron carbide in the CNT cavities. In contrast, no metallic lithium is observed around the CNT openings or the regions free of the iron carbides. These imply that it is the iron carbides that allure the lithium ions into the interior of the CNTs via the defected graphene layers. The DFT calculations indicate that the iron carbides are more lithiophilic than the graphene, explaining why the lithium ions are not intercepted with the carbon layers on their way to the cavity. With the high lithiophilicity of the iron carbides inside the CNTs, the lithium ions can reach the CNT cavity as long as there are some defects as the entrance to penetrate the graphene layer and easily reduced to lithium atoms.

Despite the similar calculations suggesting the even stronger adsorption of the iron carbides to the sodium ions than to the lithium ions, no metallic sodium is found within the CNT cavities, according to the cryo-TEM image (Figures 5D-F). This is attributed to the forbidden entrance of the sodium ions into the graphene interlayer of the CNTs. This turns out to be another condition for the deposition of the metals in the cavities: the metal ions are able to enter the interlayer and transport across the graphene.

This study proves the feasibility of metal storage in the CNT cavities and shows three prerequisites for such lithium storage 

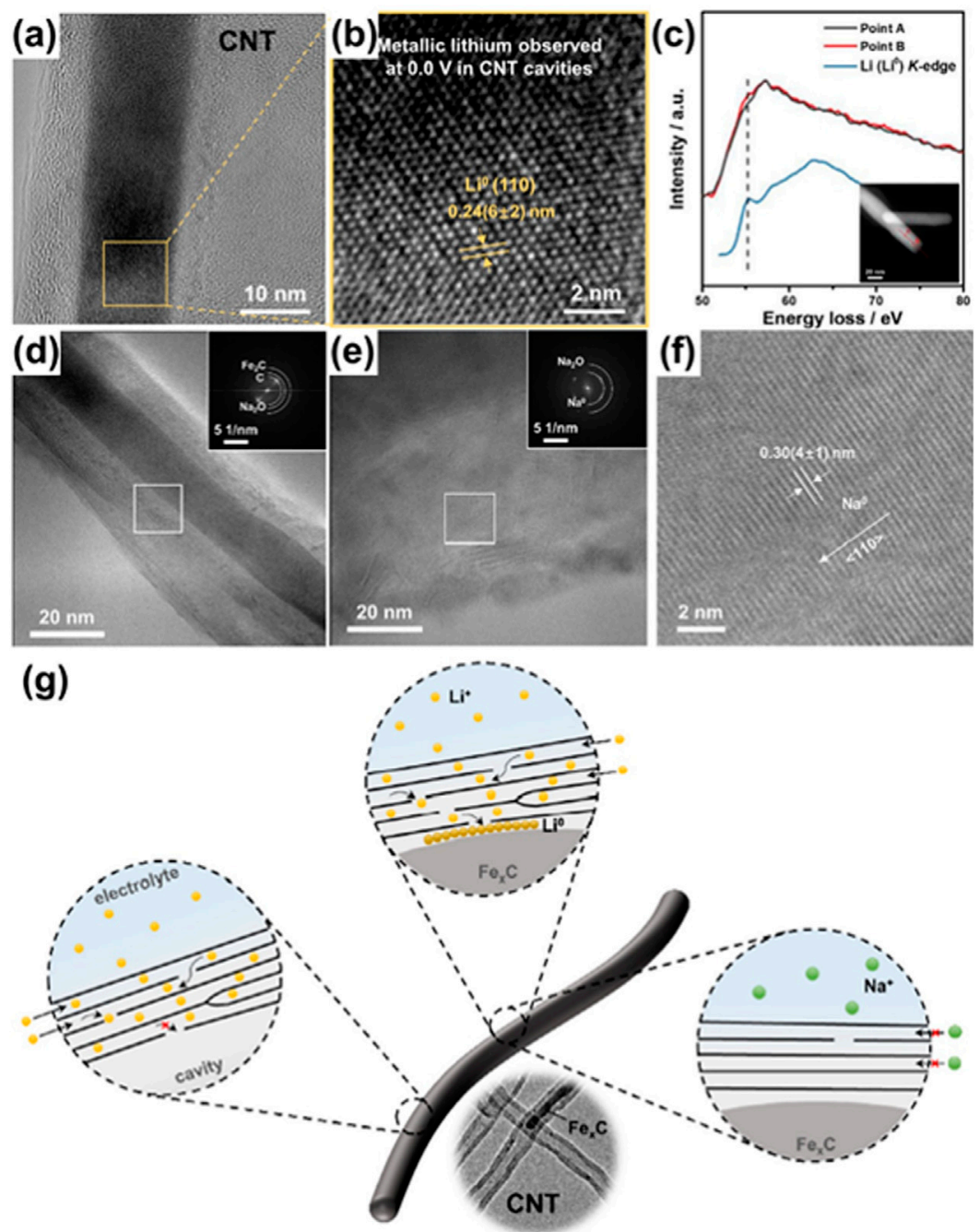

FIGURE 5 | The cryo-TEM images (A), the magnified views of the squares (B) and EELS line-scan spectra (C) of the CNTs discharged to 0.0 V. The cryo-TEM images of the CNTs (D) and metallic sodium (E), and the magnified view of the white square in e (F). Schematic comparison of the transportation of the lithium/sodium ions in the multi-walled CNT and the formation mechanism of the metallic lithium in the CNT cavities (G). Reproduced from Yang et al. (2021a).

(Figure 5G): the capability of the ions to diffuse into the cavities, the appropriate defects that help the intercalated ions to pass across the graphene layers, and the attracting species in the nano-cavities that allure the ions. These three prerequisites provide a guidance to the design of the porous carbon for an excess capacity in the cavities or nanopores.

The porous materials and "hollow" materials (such as the hollow carbon spheres, multi-walled carbon nanotubes, and fullerenes) can work as lithium containers by introducing appropriate alluring agents inside and making some defects on their shells. Similarly, other interesting species can be stored if appropriate allures can be introduced during or after the preparation of the porous host-material, not limited to the carbon materials. In this way, the composite material will present new physical and chemical properties and find new applications, such as new catalysts and high-capacity electrode materials.

\section{CONCLUSION AND OUTLOOK}

Recent years have seen significant advances in realizing controlled lithium deposition by designing proper current collectors. Researchers conducted plenty of studies on designing the structures and selecting the materials of the current collector. Although some of these approaches can achieve dendrite-free lithium deposition, it should be acknowledged that there is still a long way to go before the commercial application of the secondary lithium metal batteries. Further studies may be focused on the following aspects:

1. In-depth study of the relationship between the lithium plating/ stripping behaviors and the properties of substrate materials. At present, a wide range of materials have been applied as current collector and have shown improved electrochemical behaviors. However, theoretical studies of lithium deposition 
behaviors on the current collectors as well as fundamental understandings of how the current collectors affect the lithium metal nucleation and growth are limited and further probing is required. Our preliminary studies indicate that the electrochemical behaviors of the lithiophilic (non)metals can be predicted with the phase diagram. However, it is undeniable that some other factors such as the hardness, wettability, surface diffusion activation energy of lithium, lithium adsorption energy, and surface morphology of the substrate materials affect the lithium deposition and should be taken into account for further investigation.

2. Optimization of the $3 \mathrm{D}$ porous current collector. Although it has been clearly demonstrated that these $3 \mathrm{D}$ porous current collectors are able to suppress the dendrite growth by guiding the lithium deposition and reduce the volume change, there are still some challenges to overcome to achieve a commercialized lithium metal anode, for example, severe side reactions and low Coulombic efficiencies resulted from the high specific surface area, low volumetric energy density of the full cell, and the instability of some fine designed micro/ nano structures during repeated cycles.

3. Development of composite anodes. In practical applications, it is necessary to pre-store lithium metal in the current collector, especially for the Li-S and $\mathrm{Li}-\mathrm{O}_{2}$ batteries. Thermal infusion is a common method for preloading lithium into the $3 \mathrm{D}$ porous current collectors to prepare composite electrodes. Its prerequisite is the thermal stability and wettability of liquid lithium to the substrate material. It is beneficial to the preparation of composite anodes by designing a lithiophilic interface layer on the surface of a $3 \mathrm{D}$ current collector, or directly using a lithiophilic material as the $3 \mathrm{D}$ current collector. The composite anode can maintain the characteristics of a $3 \mathrm{D}$ porous structure, and can also adjust the lithium loading simply by controlling the processing time.

4. Applications of current collectors doped with a marginal amount of lithiophilic metals. The lithiophilic alloys can be used to reduce the nucleation overpotential of lithium, but introduction of a large number of alloying elements reduces the energy density of the battery, and the large volume changes during repeated alloying/de-alloying may damage the integrity of the substrate. Therefore, lithium doped with trace lithiophilic metal elements is promising as an alternative anode for pure lithium metal. Doping a trace amount of $\mathrm{Ag}, \mathrm{Al}$ or $\mathrm{Mg}(<2 \mathrm{at} \%)$ in lithium foil can significantly change the surface properties of the lithium metal, such as

\section{REFERENCES}

An, Y., Fei, H., Zeng, G., Xu, X., Ci, L., Xi, B., et al. (2018). Vacuum Distillation Derived 3D Porous Current Collector for Stable Lithium-Metal Batteries. Nano Energy 47, 503-511. doi:10.1016/j.nanoen.2018.03.036

Chen, K.-H., Sanchez, A. J., Kazyak, E., Davis, A. L., and Dasgupta, N. P. (2019a). Synergistic Effect of 3D Current Collectors and ALD Surface Modification for High Coulombic Efficiency Lithium Metal Anodes. Adv. Energ. Mater. 9 (4), 1802534. doi:10.1002/aenm.201802534

Chen, L., Fan, X., Ji, X., Chen, J., Hou, S., and Wang, C. (2019b). High-Energy Li Metal Battery with Lithiated Host. Joule 3 (3), 732-744. doi:10.1016/j.joule.2018.11.025 surface energy, (Choi et al., 2019), lithium adsorption energy, (Wu et al., 2020), chemical reactivity.(Lu et al., 2020). This kind of current collector can be manufactured on a large scale, which provides favorable support for its potential industrialization.

5. Design of the lithiophilic-lithiophobic gradient 3D frameworks. The $\mathrm{Li}^{+}$transport resistance varies within the 3D structure of the current collector according to the distance between the Li deposition spots and the cathode. Lithium is preferentially deposited on the top of the 3D framework, due to the short lithium ion transport pathway, which reduces the utilization of the 3D framework. By arranging the positions of the lithiophilic/lithiophobic materials, the lithium deposition can be more effectively adjusted. For example, the lithiophilic $\mathrm{Au}$ particles can be plated on the bottom of the carbon materials (Xiang et al., 2018; Pu et al., 2019) and the lithiophobic layered metal-organic framework nanosheets can be placed on the top of the 3D framework (Noh et al., 2021) (near the separator) to construct the lithiophiliclithiophobic gradient 3D framework. The gradient 3D framework can guide homogeneous, bottom-up $\mathrm{Li}$ deposition and top-down $\mathrm{Li}$ dissolution and decrease the formation of lithium dendrite.

6. Combination of different strategies. Each strategy has its own features and limitations. Consequently, no single strategy can satisfy the needs of the practical applications of lithium metal batteries. For instance, the introduction of the artificial SEI layer can further inhibit the side reactions on the $3 \mathrm{D}$ current collector/electrolyte interface to improve the Coulomb efficiency. Such work have been in progress in this group and will be reviewed later.

\section{AUTHOR CONTRIBUTIONS}

SZ wrote the manuscript draft and made the figures. ZW and LC conceptualized, wrote the manuscript draft, and corrected the manuscript. GY, XL and YL corrected the manuscript. All authors contributed to the article and approved the submitted version.

\section{FUNDING}

This work was financially supported by the National Natural Science Foundation of China (NSFC No. 21773301).

Chen, X., Chen, X.-R., Hou, T.-Z., Li, B.-Q., Cheng, X.-B., Zhang, R., et al. (2019c). Lithiophilicity Chemistry of Heteroatom-Doped Carbon to Guide Uniform Lithium Nucleation in Lithium Metal Anodes. Sci. Adv. 5 (2), eaau7728. doi:10. 1126/sciadv.aau7728

Cheng, X.-B., Hou, T.-Z., Zhang, R., Peng, H.-J., Zhao, C.-Z., Huang, J.-Q., et al. (2016). Dendrite-Free Lithium Deposition Induced by Uniformly Distributed Lithium Ions for Efficient Lithium Metal Batteries. Adv. Mater. 28 (15), 2888-2895. doi:10.1002/adma.201506124

Cheng, X.-B., Peng, H.-J., Huang, J.-Q., Zhang, R., Zhao, C.-Z., and Zhang, Q. (2015). Dual-Phase Lithium Metal Anode Containing a Polysulfide-Induced Solid Electrolyte Interphase and Nanostructured Graphene Framework for LithiumSulfur Batteries. ACS Nano 9 (6), 6373-6382. doi:10.1021/acsnano.5b01990 
Cheng, X.-B., Zhang, R., Zhao, C.-Z., and Zhang, Q. (2017). Toward Safe Lithium Metal Anode in Rechargeable Batteries: A Review. Chem. Rev. 117 (15), 10403-10473. doi:10.1021/acs.chemrev.7b00115

Choi, S. H., Lee, S. J., Yoo, D. J., Park, J. H., Park, J. H., Ko, Y. N., et al. (2019). Marginal Magnesium Doping for High-Performance Lithium Metal Batteries. Adv. Energ. Mater. 9 (41), 1902278. doi:10.1002/aenm.201902278

Corsi, J. S., Welborn, S. S., Stach, E. A., and Detsi, E. (2021). Insights into the Degradation Mechanism of Nanoporous Alloy-Type Li-Ion Battery Anodes. ACS Energ. Lett. 6 (5), 1749-1756. doi:10.1021/acsenergylett.1c00324

Deng, W., Zhu, W., Zhou, X., and Liu, Z. (2018). Graphene Nested Porous Carbon Current Collector for Lithium Metal Anode with Ultrahigh Areal Capacity. Energ. Storage Mater. 15, 266-273. doi:10.1016/j.ensm.2018.05.005

Dong, H., Xiao, X., Jin, C., Wang, X., Tang, P., Wang, C., et al. (2019). High Lithium-Ion Conductivity Polymer Film to Suppress Dendrites in Li Metal Batteries. J. Power Sourc. 423, 72-79. doi:10.1016/j.jpowsour.2019.03.032

Dong, P., Zhang, X., Cha, Y., Lee, J.-I., and Song, M.-K. (2020). In Situ surface protection of Lithium Metal Anode in Lithium-Selenium Disulfide Batteries with Ionic Liquid-Based Electrolytes. Nano Energy 69, 104434. doi:10.1016/j. nanoen.2019.104434

Gao, P., Wu, H., Zhang, X., Jia, H., Kim, J. M., Engelhard, M. H., et al. (2021). Optimization of Magnesium-Doped Lithium Metal Anode for High Performance Lithium Metal Batteries through Modeling and Experiment. Angew. Chem. 133 (30), 16642-16649. doi:10.1002/anie.20210334410.1002/ ange.202103344

Guo, F., Kang, T., Liu, Z., Tong, B., Guo, L., Wang, Y., et al. (2019). Advanced Lithium Metal-Carbon Nanotube Composite Anode for High-Performance Lithium-Oxygen Batteries. Nano Lett. 19 (9), 6377-6384. doi:10.1021/acs. nanolett.9b02560

Guo, F., Wang, Y., Kang, T., Liu, C., Shen, Y., Lu, W., et al. (2018). A Li-Dual Carbon Composite as Stable Anode Material for Li Batteries. Energ. Storage Mater. 15, 116-123. doi:10.1016/j.ensm.2018.03.018

Hou, T., Yang, G., Rajput, N. N., Self, J., Park, S.-W., Nanda, J., et al. (2019). The Influence of FEC on the Solvation Structure and Reduction Reaction of LiPF6/ EC Electrolytes and its Implication for Solid Electrolyte Interphase Formation. Nano Energy 64, 103881. doi:10.1016/j.nanoen.2019.103881

Hu, Y., Qiao, Y., Xie, Z., Li, L., Qu, M., Liu, W., et al. (2021). Water-Soluble Polymer Assists Multisize Three-Dimensional Microspheres as a High-Performance Si Anode for Lithium-Ion Batteries. ACS Appl. Energ. Mater. 4 (9), 9673-9681. doi:10.1021/acsaem.1c01791

Huang, K., Li, Z., Xu, Q., Liu, H., Li, H., and Wang, Y. (2019). Lithiophilic CuO Nanoflowers on Ti-Mesh Inducing Lithium Lateral Plating Enabling Stable Lithium-Metal Anodes with Ultrahigh Rates and Ultralong Cycle Life. Adv. Energ. Mater. 9 (29), 1900853. doi:10.1002/aenm.201900853

Huang, S., Chen, L., Wang, T., Hu, J., Zhang, Q., Zhang, H., et al. (2021). SelfPropagating Enabling High Lithium Metal Utilization Ratio Composite Anodes for Lithium Metal Batteries. Nano Lett. 21 (1), 791-797. doi:10.1021/acs. nanolett.0c04546

Jiang, L. L., Yan, C., Yao, Y. X., Cai, W., Huang, J. Q., and Zhang, Q. (2021). Inhibiting Solvent Co-Intercalation in a Graphite Anode by a Localized HighConcentration Electrolyte in Fast-Charging Batteries. Angew. Chem. Int. Ed. 60 (7), 3402-3406. doi:10.1002/anie.202009738

Jiang, Y., Wang, Z., Xu, C., Li, W., Li, Y., Huang, S., et al. (2020). Atomic Layer Deposition for Improved Lithiophilicity and Solid Electrolyte Interface Stability during Lithium Plating. Energ. Storage Mater. 28, 17-26. doi:10.1016/j.ensm. 2020.01.019

Jin, C., Sheng, O., Luo, J., Yuan, H., Fang, C., Zhang, W., et al. (2017a). 3D Lithium Metal Embedded within Lithiophilic Porous Matrix for Stable Lithium Metal Batteries. Nano Energy 37, 177-186. doi:10.1016/j.nanoen.2017.05.015

Jin, S., Sun, Z., Guo, Y., Qi, Z., Guo, C., Kong, X., et al. (2017b). High Areal Capacity and Lithium Utilization in Anodes Made of Covalently Connected Graphite Microtubes. Adv. Mater. 29, 1700783. doi:10.1002/adma.201700783

Jing, H.-K., Kong, L.-L., Liu, S., Li, G.-R., and Gao, X.-P. (2015). Protected Lithium Anode with Porous Al2O3layer for Lithium-Sulfur Battery. J. Mater. Chem. A. 3 (23), 12213-12219. doi:10.1039/C5TA01490E

Kim, M. S., Deepika, S., Lee, S. H., Kim, M.-S., Ryu, J.-H., Lee, K.-R., et al. (2019). Enabling Reversible Redox Reactions in Electrochemical Cells Using Protected LiAl Intermetallics as Lithium Metal Anodes. Sci. Adv. 5 (10), eaax5587. doi:10. 1126/sciadv.aax5587
Kong, L., Li, B.-Q., Peng, H.-J., Zhang, R., Xie, J., Huang, J.-Q., et al. (2018). Porphyrin-Derived Graphene-Based Nanosheets Enabling Strong Polysulfide Chemisorption and Rapid Kinetics in Lithium-Sulfur Batteries. Adv. Energ. Mater. 8 (20), 1800849. doi:10.1002/aenm.201800849

Kong, L. L., Wang, L., Ni, Z. C., Liu, S., Li, G. R., and Gao, X. P. (2019). LithiumMagnesium Alloy as a Stable Anode for Lithium-Sulfur Battery. Adv. Funct. Mater. 29 (13), 1808756. doi:10.1002/adfm.201808756

Kuwata, H., Sonoki, H., Matsui, M., Matsuda, Y., and Imanishi, N. (2016). Surface Layer and Morphology of Lithium Metal Electrodes. Electrochemistry 84 (11), 854-860. doi:10.5796/electrochemistry.84.854

Kwon, H., Lee, J.-H., Roh, Y., Baek, J., Shin, D. J., Yoon, J. K., et al. (2021). An Electron-Deficient Carbon Current Collector for Anode-free Li-Metal Batteries. Nat. Commun. 12 (1), 5537. doi:10.1038/s41467-021-25848-1

Lee, D., Sun, S., Kwon, J., Park, H., Jang, M., Park, E., et al. (2020a). Copper Nitride Nanowires Printed Li with Stable Cycling for Li Metal Batteries in Carbonate Electrolytes. Adv. Mater. 32 (10), 1905573. doi:10.1002/adma.201905573

Lee, Y.-G., Fujiki, S., Jung, C., Suzuki, N., Yashiro, N., Omoda, R., et al. (2020b). High-energy Long-Cycling All-Solid-State Lithium Metal Batteries Enabled by Silver-Carbon Composite Anodes. Nat. Energ. 5 (4), 299-308. doi:10.1038/ s41560-020-0575-Z

Li, Q., Quan, B., Li, W., Lu, J., Zheng, J., Yu, X., et al. (2018). Electro-plating and Stripping Behavior on Lithium Metal Electrode with Ordered ThreeDimensional Structure. Nano Energy 45, 463-470. doi:10.1016/j.nanoen. 2018.01.019

Li, W., Yu, J., Wen, J., Liao, J., Ye, Z., Zhao, B., et al. (2019a). An Amorphous Zn-P/ graphite Composite with Chemical Bonding for Ultra-reversible Lithium Storage. J. Mater. Chem. A. 7 (28), 16785-16792. doi:10.1039/c9ta01431d

Li, X., Yang, G., Zhang, S., Wang, Z., and Chen, L. (2019b). Improved Lithium Deposition on Silver Plated Carbon Fiber Paper. Nano Energy 66, 104144. doi:10.1016/j.nanoen.2019.104144

Li, Y., Jiao, J., Bi, J., Wang, X., Wang, Z., and Chen, L. (2017). Controlled Deposition of Li Metal. Nano Energy 32, 241-246. doi:10.1016/j.nanoen. 2016.12.030

Li, Z., He, Q., Zhou, C., Li, Y., Liu, Z., Hong, X., et al. (2021). Rationally Design Lithiophilic Surfaces toward High-energy Lithium Metal Battery. Energ. Storage Mater. 37, 40-46. doi:10.1016/j.ensm.2021.01.012

Liao, Y., Yuan, L., Xiang, J., Zhang, W., Cheng, Z., He, B., et al. (2020). Realizing Both High Gravimetric and Volumetric Capacities in Li/3D Carbon Composite Anode. Nano Energy 69, 104471. doi:10.1016/j.nanoen.2020.104471

Lin, D., Liu, Y., Liang, Z., Lee, H.-W., Sun, J., Wang, H., et al. (2016). Layered Reduced Graphene Oxide with Nanoscale Interlayer Gaps as a Stable Host for Lithium Metal Anodes. Nat. Nanotech 11 (7), 626-632. doi:10.1038/nnano. 2016.32

Lin, K., Qin, X., Liu, M., Xu, X., Liang, G., Wu, J., et al. (2019). Ultrafine Titanium Nitride Sheath Decorated Carbon Nanofiber Network Enabling Stable Lithium Metal Anodes. Adv. Funct. Mater. 29 (46), 1903229. doi:10.1002/adfm. 201903229

Lin, S., and Zhao, J. (2020). Functional Electrolyte of Fluorinated Ether and Ester for Stabilizing Both 4.5 V LiCoO2 Cathode and Lithium Metal Anode. ACS Appl. Mater. Inter. 12 (7), 8316-8323. doi:10.1021/acsami.9b21679

Liu, F., Xu, R., Hu, Z., Ye, S., Zeng, S., Yao, Y., et al. (2019a). Regulating Lithium Nucleation via CNTs Modifying Carbon Cloth Film for Stable Li Metal Anode. Small 15 (5), 1803734. doi:10.1002/smll.201803734

Liu, L., Yin, Y.-X., Li, J.-Y., Li, N.-W., Zeng, X.-X., Ye, H., et al. (2017). FreeStanding Hollow Carbon Fibers as High-Capacity Containers for Stable Lithium Metal Anodes. Joule 1 (3), 563-575. doi:10.1016/j.joule.2017. 06.004

Liu, Q., Zhou, S., Tang, C., Zhai, Q., Zhang, X., and Wang, R. (2018a). Li-B Alloy as an Anode Material for Stable and Long Life Lithium Metal Batteries. Energies 11 (10), 2512. doi:10.3390/en11102512

Liu, S., Deng, L., Guo, W., Zhang, C., Liu, X., and Luo, J. (2019b). Bulk Nanostructured Materials Design for Fracture-Resistant Lithium Metal Anodes. Adv. Mater. 31 (15), 1807585. doi:10.1002/adma.201807585

Liu, S., Wang, A., Li, Q., Wu, J., Chiou, K., Huang, J., et al. (2018b). Crumpled Graphene Balls Stabilized Dendrite-free Lithium Metal Anodes. Joule 2 (1), 184-193. doi:10.1016/j.joule.2017.11.004

Liu, W., Xia, Y., Wang, W., Wang, Y., Jin, J., Chen, Y., et al. (2019c). Pristine or Highly Defective? Understanding the Role of Graphene Structure for Stable 
Lithium Metal Plating. Adv. Energ. Mater. 9 (3), 1802918. doi:10.1002/aenm. 201802918

Lu, K., Xu, H., He, H., Gao, S., Li, X., Zheng, C., et al. (2020). Modulating Reactivity and Stability of Metallic Lithium via Atomic Doping. J. Mater. Chem. A. 8 (20), 10363-10369. doi:10.1039/D0TA02176H

Lu, L.-L., Ge, J., Yang, J.-N., Chen, S.-M., Yao, H.-B., Zhou, F., et al. (2016). FreeStanding Copper Nanowire Network Current Collector for Improving Lithium Anode Performance. Nano Lett. 16 (7), 4431-4437. doi:10.1021/acs.nanolett. 6b01581

Lu, L.-L., Zhang, Y., Pan, Z., Yao, H.-B., Zhou, F., and Yu, S.-H. (2017). Lithiophilic $\mathrm{Cu}$-Ni Core-Shell Nanowire Network as a Stable Host for Improving Lithium Anode Performance. Energ. Storage Mater. 9, 31-38. doi:10.1016/j.ensm.2017. 06.004

Ma, X., Liu, Z., and Chen, H. (2019). Facile and Scalable Electrodeposition of Copper Current Collectors for High-Performance Li-Metal Batteries. Nano Energy 59, 500-507. doi:10.1016/j.nanoen.2019.02.048

May, R., Fritzsching, K. J., Livitz, D., Denny, S. R., and Marbella, L. E. (2021). Rapid Interfacial Exchange of Li Ions Dictates High Coulombic Efficiency in Li Metal Anodes. ACS Energ. Lett. 6 (4), 1162-1169. doi:10.1021/acsenergylett.1c00112

Niu, C., Pan, H., Xu, W., Xiao, J., Zhang, J.-G., Luo, L., et al. (2019). Self-smoothing Anode for Achieving High-Energy Lithium Metal Batteries under Realistic Conditions. Nat. Nanotechnol. 14 (6), 594-601. doi:10.1038/s41565-019-0427-9

Noh, J., Chen, W., Wu, P., Huang, Y., Tan, J., Zhou, H. C., et al. (2021). Large Cumulative Capacity Enabled by Regulating Lithium Plating with MetalOrganic Framework Layers on Porous Carbon Nanotube Scaffolds. Adv. Funct. Mater. 31 (43), 2104899. doi:10.1002/adfm.202104899

Park, J., Jeong, J., Lee, Y., Oh, M., Ryou, M.-H., and Lee, Y. M. (2016). MicroPatterned Lithium Metal Anodes with Suppressed Dendrite Formation for Post Lithium-Ion Batteries. Adv. Mater. Inter. 3 (11), 1600140. doi:10.1002/admi. 201600140

Pathak, R., Chen, K., Gurung, A., Reza, K. M., Bahrami, B., Pokharel, J., et al. (2020). Fluorinated Hybrid Solid-Electrolyte-Interphase for Dendrite-free Lithium Deposition. Nat. Commun. 11 (1), 93. doi:10.1038/s41467-01913774-2

Peng, Z., Song, J., Huai, L., Jia, H., Xiao, B., Zou, L., et al. (2019). Enhanced Stability of Li Metal Anodes by Synergetic Control of Nucleation and the Solid Electrolyte Interphase. Adv. Energ. Mater. 9 (42), 1901764. doi:10.1002/ aenm.201901764

Peng, Z., Zhao, N., Zhang, Z., Wan, H., Lin, H., Liu, M., et al. (2017). Stabilizing Li/ electrolyte Interface with a Transplantable Protective Layer Based on Nanoscale LiF Domains. Nano Energy 39, 662-672. doi:10.1016/j.nanoen.2017.07.052

Piao, N., Liu, S., Zhang, B., Ji, X., Fan, X., Wang, L., et al. (2021). Lithium Metal Batteries Enabled by Synergetic Additives in Commercial Carbonate Electrolytes. ACS Energ. Lett. 6 (5), 1839-1848. doi:10.1021/acsenergylett. $1 \mathrm{c} 00365$

Pu, J., Li, J., Zhang, K., Zhang, T., Li, C., Ma, H., et al. (2019). Conductivity and Lithiophilicity Gradients Guide Lithium Deposition to Mitigate Short Circuits. Nat. Commun. 10 (1), 1896. doi:10.1038/s41467-019-09932-1

Qiu, H., Tang, T., Asif, M., Huang, X., and Hou, Y. (2019). 3D Porous Cu Current Collectors Derived by Hydrogen Bubble Dynamic Template for Enhanced Li Metal Anode Performance. Adv. Funct. Mater. 29 (19), 1808468. doi:10.1002/ adfm.201808468

Ren, F., Peng, Z., Wang, M., Xie, Y., Li, Z., Wan, H., et al. (2019). Over-potential Induced Li/Na Filtrated Depositions Using Stacked Graphene Coating on Copper Scaffold. Energ. Storage Mater. 16, 364-373. doi:10.1016/j.ensm. 2018.06.012

Ryou, M.-H., Lee, Y. M., Lee, Y., Winter, M., and Bieker, P. (2015). Mechanical Surface Modification of Lithium Metal: Towards Improved Li Metal Anode Performance by Directed Li Plating. Adv. Funct. Mater. 25 (6), 834-841. doi:10. 1002/adfm.201402953

Shang, H., Gu, Y., Wang, Y., and Zuo, Z. (2020). N-Doped Graphdiyne Coating for Dendrite-Free Lithium Metal Batteries. Chem. Eur. J. 26, 5434-5440. doi:10. 1002/chem.201905618

Shen, F., Zhang, F., Zheng, Y., Fan, Z., Li, Z., Sun, Z., et al. (2018). Direct Growth of 3D Host on Cu Foil for Stable Lithium Metal Anode. Energ. Storage Mater. 13, 323-328. doi:10.1016/j.ensm.2018.02.005

Shi, Y., Wang, Z., Gao, H., Niu, J., Ma, W., Qin, J., et al. (2019). A Self-Supported, Three-Dimensional Porous Copper Film as a Current Collector for Advanced
Lithium Metal Batteries. J. Mater. Chem. A. 7 (3), 1092-1098. doi:10.1039/ c8ta09384a

Sun, C., Li, Y., Jin, J., Yang, J., and Wen, Z. (2019a). ZnO Nanoarray-Modified Nickel Foam as a Lithiophilic Skeleton to Regulate Lithium Deposition for Lithium-Metal Batteries. J. Mater. Chem. A. 7 (13), 7752-7759. doi:10.1039/ c9ta00862d

Sun, C., Lin, A., Li, W., Jin, J., Sun, Y., Yang, J., et al. (2019b). In Situ Conversion of $\mathrm{Cu} 3 \mathrm{P}$ Nanowires to Mixed Ion/Electron-Conducting Skeleton for Homogeneous Lithium Deposition. Adv. Energ. Mater. 10, 1902989. doi:10. 1002/aenm.201902989

Sun, Y., Zheng, G., Seh, Z. W., Liu, N., Wang, S., Sun, J., et al. (2016). GraphiteEncapsulated Li-Metal Hybrid Anodes for High-Capacity Li Batteries. Chem 1 (2), 287-297. doi:10.1016/j.chempr.2016.07.009

Tan, D. H. S., Chen, Y.-T., Yang, H., Bao, W., Sreenarayanan, B., Doux, J.-M., et al. (2021). Carbon-free High-Loading Silicon Anodes Enabled by Sulfide Solid Electrolytes. Science 373 (6562), 1494-1499. doi:10.1126/science.abg7217

Tang, W., Yin, X., Kang, S., Chen, Z., Tian, B., Teo, S. L., et al. (2018). Lithium Silicide Surface Enrichment: A Solution to Lithium Metal Battery. Adv. Mater. 30 (34), 1801745. doi:10.1002/adma.201801745

Tarascon, J.-M., and Armand, M. (2001). Issues and Challenges Facing Rechargeable Lithium Batteries. Nature 414 (6861), 359-367. doi:10.1038/ 35104644

Wahyudi, W., Ladelta, V., Tsetseris, L., Alsabban, M. M., Guo, X., Yengel, E., et al. (2021). Lithium-Ion Desolvation Induced by Nitrate Additives Reveals New Insights into High Performance Lithium Batteries. Adv. Funct. Mater. 31 (23), 2101593. doi:10.1002/adfm.202101593

Wan, M., Kang, S., Wang, L., Lee, H.-W., Zheng, G. W., Cui, Y., et al. (2020). Mechanical Rolling Formation of Interpenetrated Lithium Metal/lithium Tin alloy Foil for Ultrahigh-Rate Battery Anode. Nat. Commun. 11 (1), 829. doi:10. 1038/s41467-020-14550-3

Wang, A., Zhang, X., Yang, Y.-W., Huang, J., Liu, X., and Luo, J. (2018a). Horizontal Centripetal Plating in the Patterned Voids of Li/Graphene Composites for Stable Lithium-Metal Anodes. Chem 4 (9), 2192-2200. doi:10.1016/j.chempr.2018.06.017

Wang, S.-H., Yin, Y.-X., Zuo, T.-T., Dong, W., Li, J.-Y., Shi, J.-L., et al. (2017a). Stable Li Metal Anodes via Regulating Lithium Plating/Stripping in Vertically Aligned Microchannels. Adv. Mater. 29, 1703729. doi:10.1002/adma. 201703729

Wang, X., Pan, Z., Wu, Y., Ding, X., Hong, X., Xu, G., et al. (2019). Infiltrating Lithium into Carbon Cloth Decorated with Zinc Oxide Arrays for Dendrite-free Lithium Metal Anode. Nano Res. 12 (3), 525-529. doi:10.1007/s12274-0182245-Z

Wang, Y., Shen, Y., Du, Z., Zhang, X., Wang, K., Zhang, H., et al. (2017b). A Lithium-Carbon Nanotube Composite for Stable Lithium Anodes. J. Mater. Chem. A. 5 (45), 23434-23439. doi:10.1039/C7TA08531A

Wang, Y., Wang, Z., Lei, D., Lv, W., Zhao, Q., Ni, B., et al. (2018b). Spherical Li Deposited inside 3D Cu Skeleton as Anode with Ultrastable Performance. ACS Appl. Mater. Inter. 10 (24), 20244-20249. doi:10.1021/acsami.8b04881

Wei, L., Li, L., Zhao, T., Zhang, N., Zhao, Y., Wu, F., et al. (2020). MOF-derived Lithiophilic CuO Nanorod Arrays for Stable Lithium Metal Anodes. Nanoscale 12 (17), 9416-9422. doi:10.1039/D0NR01091J

Wu, C., Huang, H., Lu, W., Wei, Z., Ni, X., Sun, F., et al. (2020). Mg Doped Li-LiB Alloy with In Situ Formed Lithiophilic LiB Skeleton for Lithium Metal Batteries. Adv. Sci. 7 (6), 1902643. doi:10.1002/advs.201902643

Xiang, J., Yuan, L., Shen, Y., Cheng, Z., Yuan, K., Guo, Z., et al. (2018). Improved Rechargeability of Lithium Metal Anode via Controlling Lithium-Ion Flux. Adv. Energ. Mater. 8 (36), 1802352. doi:10.1002/ aenm. 201802352

Xu, Q., Lin, J., Ye, C., Jin, X., Ye, D., Lu, Y., et al. (2020a). Air-Stable and DendriteFree Lithium Metal Anodes Enabled by a Hybrid Interphase of C 60 and Mg. Adv. Energ. Mater. 10 (6), 1903292. doi:10.1002/aenm.201903292

Xu, W., Wang, J., Ding, F., Chen, X., Nasybulin, E., Zhang, Y., et al. (2014). Lithium Metal Anodes for Rechargeable Batteries. Energy Environ. Sci. 7 (2), 513-537. doi:10.1039/c3ee40795k

Xu, Y., Wu, H., He, Y., Chen, Q., Zhang, J.-G., Xu, W., et al. (2020b). Atomic to Nanoscale Origin of Vinylene Carbonate Enhanced Cycling Stability of Lithium Metal Anode Revealed by Cryo-Transmission Electron Microscopy. Nano Lett. 20 (1), 418-425. doi:10.1021/acs.nanolett.9b04111 
Xue, P., Liu, S., Shi, X., Sun, C., Lai, C., Zhou, Y., et al. (2018). A Hierarchical SilverNanowire-Graphene Host Enabling Ultrahigh Rates and Superior Long-Term Cycling of Lithium-Metal Composite Anodes. Adv. Mater. 30 (44), 1804165. doi:10.1002/adma.201804165

Yan, C., Cheng, X.-B., Yao, Y.-X., Shen, X., Li, B.-Q., Li, W.-J., et al. (2018a). An Armored Mixed Conductor Interphase on a Dendrite-free LithiumMetal Anode. Adv. Mater. 30 (43), 1804461-1814059. doi:10.1002/adma. 201804461

Yan, K., Lu, Z., Lee, H.-W., Xiong, F., Hsu, P.-C., Li, Y., et al. (2016). Selective Deposition and Stable Encapsulation of Lithium through Heterogeneous Seeded Growth. Nat. Energ. 1, 16010. doi:10.1038/nenergy.2016.10

Yan, K., Sun, B., Munroe, P., and Wang, G. (2018b). Three-dimensional Pie-like Current Collectors for Dendrite-free Lithium Metal Anodes. Energ. Storage Mater. 11, 127-133. doi:10.1016/j.ensm.2017.10.012

Yang, C.-P., Yin, Y.-X., Zhang, S.-F., Li, N.-W., and Guo, Y.-G. (2015). Accommodating Lithium into 3D Current Collectors with a Submicron Skeleton towards Long-Life Lithium Metal Anodes. Nat. Commun. 6, 8058. doi:10.1038/ncomms9058

Yang, C., Yao, Y., He, S., Xie, H., Hitz, E., and Hu, L. (2017). Ultrafine Silver Nanoparticles for Seeded Lithium Deposition toward Stable Lithium Metal Anode. Adv. Mater. 29, 1702714. doi:10.1002/adma.201702714

Yang, G., Li, Y., Liu, S., Zhang, S., Wang, Z., and Chen, L. (2019a). LiFSI to Improve Lithium Deposition in Carbonate Electrolyte. Energ. Storage Mater. 23, 350-357. doi:10.1016/j.ensm.2019.04.041

Yang, G., Li, Y., Tong, Y., Qiu, J., Liu, S., Zhang, S., et al. (2019b). Lithium Plating and Stripping on Carbon Nanotube Sponge. Nano Lett. 19 (1), 494-499. doi:10. 1021/acs.nanolett.8b04376

Yang, G., Liu, Z., Weng, S., Zhang, Q., Wang, X., Wang, Z., et al. (2021a). Iron Carbide Allured Lithium Metal Storage in Carbon Nanotube Cavities. Energ. Storage Mater. 36, 459-465. doi:10.1016/j.ensm.2021.03.01810.1016/j.ensm. 2021.01.022

Yang, G., Zhang, S., Tong, Y., Li, X., Wang, Z., and Chen, L. (2019c). Minimizing Carbon Particle Size to Improve Lithium Deposition on Natural Graphite. Carbon 155, 9-15. doi:10.1016/j.carbon.2019.08.023

Yang, G., Zhang, S., Weng, S., Li, X., Wang, X., Wang, Z., et al. (2021b). Anionic Effect on Enhancing the Stability of a Solid Electrolyte Interphase Film for Lithium Deposition on Graphite. Nano Lett. 21 (12), 5316-5323. doi:10.1021/ acs.nanolett.1c01436

Ye, H., Zheng, Z. J., Yao, H. R., Liu, S. C., Zuo, T. T., Wu, X. W., et al. (2019a). Guiding Uniform Li Plating/Stripping through Lithium-Aluminum Alloying Medium for Long-Life Li Metal Batteries. Angew. Chem. Int. Ed. 58 (4), 1094-1099. doi:10.1002/anie.201811955

Ye, Y., Liu, Y., Wu, J., and Yang, Y. (2020). Lithiophilic Li-Zn alloy Modified 3D Cu Foam for Dendrite-free Lithium Metal Anode. J. Power Sourc. 472, 228520. doi:10.1016/j.jpowsour.2020.228520

Yu, L., Canfield, N. L., Chen, S., Lee, H., Ren, X., Engelhard, M. H., et al. (2018). Enhanced Stability of Lithium Metal Anode by Using a 3D Porous Nickel Substrate. ChemElectroChem 5 (5), 761-769. doi:10.1002/celc. 201701250

Yuan, Y., Wu, F., Chen, G., Bai, Y., and Wu, C. (2019). Porous LiF Layer Fabricated by a Facile Chemical Method toward Dendrite-free Lithium Metal Anode. J. Energ. Chem. 37, 197-203. doi:10.1016/j.jechem.2019.03.014

Yue, X.-Y., Bao, J., Yang, S.-Y., Luo, R.-J., Wang, Q.-C., Wu, X.-J., et al. (2020). Petaloid-shaped $\mathrm{ZnO}$ Coated Carbon Felt as a Controllable Host to Construct Hierarchical Li Composite Anode. Nano Energy 71, 104614. doi:10.1016/j. nanoen.2020.104614

Yue, X.-Y., Wang, W.-W., Wang, Q.-C., Meng, J.-K., Zhang, Z.-Q., Wu, X.-J., et al. (2018). CoO Nanofiber Decorated Nickel Foams as Lithium Dendrite Suppressing Host Skeletons for High Energy Lithium Metal Batteries. Energ. Storage Mater. 14, 335-344. doi:10.1016/j.ensm.2018.05.017

Yue, X. Y., Zhou, Q. Y., Bao, J., Ma, C., Yang, S. Y., Li, X. L., et al. (2021). In Situ Construction of Lithium Silicide Host with Unhindered Lithium Spread for Dendrite-Free Lithium Metal Anode. Adv. Funct. Mater. 31 (9), 2008786. doi:10.1002/adfm.202008786

Yun, Q., He, Y.-B., Lv, W., Zhao, Y., Li, B., Kang, F., et al. (2016). Chemical Dealloying Derived 3D Porous Current Collector for Li Metal Anodes. Adv. Mater. 28 (32), 6932-6939. doi:10.1002/adma.201601409
Zhai, P., Wei, Y., Xiao, J., Liu, W., Zuo, J., Gu, X., et al. (2020). In Situ Generation of Artificial Solid-Electrolyte Interphases on 3D Conducting Scaffolds for HighPerformance Lithium-Metal Anodes. Adv. Energ. Mater. 10 (8), 1903339. doi:10.1002/aenm.201903339

Zhang, C., Lv, W., Zhou, G., Huang, Z., Zhang, Y., Lyu, R., et al. (2018a). Vertically Aligned Lithiophilic $\mathrm{CuO}$ Nanosheets on a $\mathrm{Cu}$ Collector to Stabilize Lithium Deposition for Lithium Metal Batteries. Adv. Energ. Mater. 8 (21), 1703404. doi:10.1002/aenm.201703404

Zhang, C., Lyu, R., Lv, W., Li, H., Jiang, W., Li, J., et al. (2019a). A Lightweight 3D $\mathrm{Cu}$ Nanowire Network with Phosphidation Gradient as Current Collector for High-Density Nucleation and Stable Deposition of Lithium. Adv. Mater. 31 (48), 1904991. doi:10.1002/adma.201904991

Zhang, D., Dai, A., Wu, M., Shen, K., Xiao, T., Hou, G., et al. (2020a). Lithiophilic 3D Porous CuZn Current Collector for Stable Lithium Metal Batteries. ACS Energ. Lett. 5 (1), 180-186. doi:10.1021/acsenergylett.9b01987

Zhang, Q., Luan, J., Tang, Y., Ji, X., Wang, S., and Wang, H. (2018b). A Facile Annealing Strategy for Achieving In Situ Controllable Cu2O Nanoparticle Decorated Copper Foil as a Current Collector for Stable Lithium Metal Anodes. J. Mater. Chem. A. 6 (38), 18444-18448. doi:10.1039/c8ta07612j

Zhang, R., Chen, X., Shen, X., Zhang, X.-Q., Chen, X.-R., Cheng, X.-B., et al. (2018c). Coralloid Carbon Fiber-Based Composite Lithium Anode for Robust Lithium Metal Batteries. Joule 2 (4), 764-777. doi:10.1016/j. joule.2018.02.001

Zhang, R., Cheng, X.-B., Zhao, C.-Z., Peng, H.-J., Shi, J.-L., Huang, J.-Q., et al. (2016). Conductive Nanostructured Scaffolds Render Low Local Current Density to Inhibit Lithium Dendrite Growth. Adv. Mater. 28 (11), 2155-2162. doi:10.1002/adma.201504117

Zhang, S., Yang, G., Liu, S., Li, X., Wang, X., Wang, Z., et al. (2020b). Understanding the Dropping of Lithium Plating Potential in Carbonate Electrolyte. Nano Energy 70, 104486. doi:10.1016/j.nanoen.2020.104486

Zhang, S., Yang, G., Liu, Z., Li, X., Wang, X., Chen, R., et al. (2021a). Competitive Solvation Enhanced Stability of Lithium Metal Anode in Dual-Salt Electrolyte. Nano Lett. 21 (7), 3310-3317. doi:10.1021/acs.nanolett.1c00848

Zhang, S., Yang, G., Liu, Z., Weng, S., Li, X., Wang, X., et al. (2021b). Phase Diagram Determined Lithium Plating/Stripping Behaviors on Lithiophilic Substrates. ACS Energ. Lett. 6 (11), 4118-4126. doi:10.1021/acsenergylett. $1 \mathrm{c} 02127$

Zhang, W., Jin, H., Xu, C., Zhao, S., Du, Y., and Zhang, J. (2019b). Diffusion Couples $\mathrm{Cu}-\mathrm{X}(\mathrm{X}=\mathrm{Sn}, \mathrm{Zn}, \mathrm{Al})$ Derived 3D Porous Current Collector for Dendrite-free Lithium Metal Battery. J. Power Sourc. 440, 227142. doi:10. 1016/j.jpowsour.2019.227142

Zhang, X.-Q., Cheng, X.-B., Chen, X., Yan, C., and Zhang, Q. (2017a). Fluoroethylene Carbonate Additives to Render Uniform Li Deposits in Lithium Metal Batteries. Adv. Funct. Mater. 27 (10), 1605989. doi:10.1002/ adfm.201605989

Zhang, X., Wang, A., Lv, R., and Luo, J. (2019c). A Corrosion-Resistant Current Collector for Lithium Metal Anodes. Energ. Storage Mater. 18, 199-204. doi:10. 1016/j.ensm.2018.09.017

Zhang, Y., Luo, W., Wang, C., Li, Y., Chen, C., Song, J., et al. (2017b). Highcapacity, Low-Tortuosity, and Channel-Guided Lithium Metal Anode. Proc. Natl. Acad. Sci. USA 114 (14), 3584-3589. doi:10.1073/pnas.1618871114

Zhang, Y., Shi, Y., Hu, X. C., Wang, W. P., Wen, R., Xin, S., et al. (2020c). A 3D Lithium/Carbon Fiber Anode with Sustained Electrolyte Contact for SolidState Batteries. Adv. Energ. Mater. 10 (3), 1903325. doi:10.1002/aenm. 201903325

Zhao, F., Zhou, X., Deng, W., and Liu, Z. (2019). Entrapping Lithium Deposition in Lithiophilic Reservoir Constructed by Vertically Aligned ZnO Nanosheets for Dendrite-free Li Metal Anodes. Nano Energy 62, 55-63. doi:10.1016/j.nanoen. 2019.04.087

Zhao, H., Lei, D., He, Y.-B., Yuan, Y., Yun, Q., Ni, B., et al. (2018). Compact 3D Copper with Uniform Porous Structure Derived by Electrochemical Dealloying as Dendrite-free Lithium Metal Anode Current Collector. Adv. Energ. Mater. 8 (19), 1800266. doi:10.1002/aenm.201800266

Zhao, J., Zhou, G., Yan, K., Xie, J., Li, Y., Liao, L., et al. (2017). Air-stable and Freestanding Lithium alloy/graphene Foil as an Alternative to Lithium Metal Anodes. Nat. Nanotech 12 (10), 993-999. doi:10.1038/nnano. 2017.129 
Zheng, H., Zhang, Q., Chen, Q., Xu, W., Xie, Q., Cai, Y., et al. (2020). 3D LithiophilicLithiophobic-Lithiophilic Dual-Gradient Porous Skeleton for Highly Stable Lithium Metal Anode. J. Mater. Chem. A. 8 (1), 313-322. doi:10.1039/C9TA09505E

Zhuang, H., Zhao, P., Li, G., Xu, Y., and Jia, X. (2020). Li-LiAl alloy Composite with Memory Effect as High-Performance Lithium Metal Anode. J. Power Sourc. 455, 227977. doi:10.1016/j.jpowsour.2020.227977

Zou, P., Wang, Y., Chiang, S.-W., Wang, X., Kang, F., and Yang, C. (2018). Directing Lateral Growth of Lithium Dendrites in Micro-compartmented Anode Arrays for Safe Lithium Metal Batteries. Nat. Commun. 9 (1), 464. doi:10.1038/s41467-018-02888-8

Zuo, T.-T., Wu, X.-W., Yang, C.-P., Yin, Y.-X., Ye, H., Li, N.-W., et al. (2017). Graphitized Carbon Fibers as Multifunctional 3D Current Collectors for High Areal Capacity Li Anodes. Adv. Mater. 29 (29), 1700389. doi:10.1002/adma.201700389

Zuo, T.-T., Yin, Y.-X., Wang, S.-H., Wang, P.-F., Yang, X., Liu, J., et al. (2018). Trapping Lithium into Hollow Silica Microspheres with a Carbon Nanotube Core for Dendrite-free Lithium Metal Anodes. Nano Lett. 18 (1), 297-301. doi:10.1021/acs.nanolett.7b04136
Conflict of Interest: The authors declare that the research was conducted in the absence of any commercial or financial relationships that could be construed as a potential conflict of interest.

Publisher's Note: All claims expressed in this article are solely those of the authors and do not necessarily represent those of their affiliated organizations, or those of the publisher, the editors and the reviewers. Any product that may be evaluated in this article, or claim that may be made by its manufacturer, is not guaranteed or endorsed by the publisher.

Copyright $\odot 2022$ Zhang, Yang, Li, Li, Wang and Chen. This is an open-access article distributed under the terms of the Creative Commons Attribution License (CC BY). The use, distribution or reproduction in other forums is permitted, provided the original author(s) and the copyright owner(s) are credited and that the original publication in this journal is cited, in accordance with accepted academic practice. No use, distribution or reproduction is permitted which does not comply with these terms. 